\title{
MONOTONE SKEW-PRODUCT SEMIFLOWS FOR CARATHÉODORY DIFFERENTIAL EQUATIONS AND APPLICATIONS
}

\author{
Dedicated to the memory of Genèvieve Raugel
}

IACOPO P. LONGO, SYLVIA NOVO, AND RAFAEL OBAYA

\begin{abstract}
The first part of the paper is devoted to studying the continuous dependence of the solutions of Carathéodory constant delay differential equations where the vector fields satisfy classical cooperative conditions. As a consequence, when the set of considered vector fields is invariant with respect to the time-translation map, the continuity of the respective induced skew-product semiflows is obtained. These results are important for the study of the long-term behavior of the trajectories. In particular, the construction of semicontinuous semiequilibria and equilibria is extended to the context of ordinary and delay Carathéodory differential equations. Under appropriate assumptions of sublinearity, the existence of a unique continuous equilibrium, whose graph coincides with the pullback attractor for the evolution processes, is shown. The conditions under which such a solution is the forward attractor of the considered problem are outlined. Two examples of application of the developed tools are also provided.
\end{abstract}

\section{INTRODUCTION}

This paper deals with the study of families of delay differential equations of the form $x^{\prime}(t)=f(t, x(t), x(t-1)), f \in E$, where $E$ is a set of Lipschitz Carathéodory functions $f: \mathbb{R} \times \mathbb{R}^{2 N} \rightarrow \mathbb{R}^{N},(t, x, y) \rightarrow f(t, x, y)$ satisfying the usual cooperative conditions in their arguments. The set of initial data is $C\left([-1,0], \mathbb{R}^{N}\right)$ endowed with the topology of the uniform convergence, which is a partially ordered Banach space with positive cone $C\left([-1,0],\left(\mathbb{R}^{+}\right)^{N}\right)$. For simplicity, these sets will be denoted by $\mathcal{C}$ and $\mathcal{C}^{+}$respectively. Adequate topologies of continuity for $E$, that is, assuring the continuity of the solutions with respect to the functions in $E$ and the initial data, will be provided.

The study of the topologies of continuity for Carathéodory ordinary differential equation is a classical question with important implications in the topic of nonautonomous differential equations and its applications. In particular, Artstein [1, 2, 3], Heunis [9], Miller and Sell [16, 17], Neustadt [18], Opial [22], Sell [24] introduced and studied strong and weak topologies of integral type on the space of Lipschitz

2020 Mathematics Subject Classification. 34K05, 34K25, 34A34, 37B55, 34A12.

Key words and phrases. Carathéodory functions, non-autonomous Carathéodory delay and ordinary differential equations, continuous dependence on initial data, monotone semiflows.

All authors were partly supported by MICIIN/FEDER project RTI2018-096523-B-100 and by the EU Marie-Skłodowska-Curie ITN grant H2020-MSCA-ITN-2014 643073. I.P. Longo was also supported by the European Unions Horizon 2020 research and innovation programme under the Marie Skodowska-Curie grant agreement No 754462. 
Carathéodory functions. The convergence of a sequence $\left(f_{n}\right)_{n \in \mathbb{N}}$ with respect to these topologies requires the convergence of the integrals of the evaluation of the functions $f_{n}$, either pointwise in $\mathbb{R}^{N}$ (topologies $\mathcal{T}_{P}$ and $\sigma_{P}$ ), or uniformly on any bounded set of continuous functions (topology $\mathcal{T}_{B}$ ). Recently, Longo et al. [13, 14] completed some parts of this theory by introducing the strong and weak topologies $T_{\Theta}$ and $\sigma_{\Theta}$, where $\Theta$ is a suitable set of moduli of continuity. When the set of the $m$-bounds of the functions in $E$ is equicontinuous, each set of uniformly bounded solutions on a compact interval admit a common modulus of continuity, and then it is possible to construct a countable set of moduli of continuity $\Theta$ such that $T_{\Theta}$ and $\sigma_{\Theta}$ become topologies of continuity for the flow map.

The extension of the previous idea to Carathéodory delay differential equations is problematic because the initial data do not generally share the same modulus of continuity of the solutions, so that the choice of a suitable set of moduli of continuity $\Theta$ is not possible. Longo et al. [15] deal with this important question and introduce the hybrid topologies $\mathcal{T}_{\Theta \hat{\Theta}}, \sigma_{\Theta} \hat{\Theta}, \mathcal{T}_{\Theta D}$ and $\sigma_{\Theta D}$, where $\Theta, \widehat{\Theta}$ are suitable sets of moduli of continuity and $D$ is a countable dense subset of $\mathbb{R}^{N}$. The term hybrid refers to the fact that these topologies are derived from the previous ones but treat the $x$ and $y$ components differently.

In this paper, the set $E$ will be endowed with one of these strong or weak hybrid topologies. However, in contrast with [15], no assumptions on the $l$-bounds of $E$ will be considered. Nevertheless, we show that such topologies are still of continuity under very general cooperative conditions. In addition, if $E$ is closed and invariant under the flow defined by the time-translation in the space of Carathéodory functions, the continuity of the induced local or global monotone skew-product semiflow in $E \times \mathcal{C}$ is deduced. As a consequence, we develop topological methods to analyze the long-term behavior of the trajectories. More precisely, tools for random and deterministic monotone dynamical systems are extended to this new situation. In particular, we define and construct semicontinuous semiequilibria providing a natural version, valid in this context, of some result of Chuesov [6], Novo et al. [19] and Zhao [29].

The structure and main results of the paper are organized as follows. In Section 2 we introduce the topological spaces which are used throughout the paper and their properties. We also include a subsection on Carathéodory differential equations with constant delay, stating a result of [15] concerning the continuous dependence of the solutions for some weak and strong hybrids topologies when the $L_{l o c}^{1}$-equicontinuity of the $m$-bounds holds.

Section 3 focuses on the monotone case, where less conditions are needed to obtain topologies of continuity, and hence for the continuity of the induced skewproduct semiflow. In particular, when the $L_{l o c}^{1}$-equicontinuity of the $m$-bounds and a monotonicity assumption $\left(\mathbf{K}_{y}\right)$ on the $y$ component are assumed, all the strong (resp. weak) hybrid topologies stated above coincide for any $\hat{\Theta}$ and any $D$, provided that $\Theta$ is the set of moduli of continuity defined by the $m$-bounds. In [15], the boundedness of the $l$-bounds was assumed instead of the monotonicity to obtain such a result. Moreover, when the monotone condition $\left(\mathbf{K}_{x y}\right)$ with respect to both $x$ and $y$ holds, then all the previous strong topologies coincide with $\mathcal{T}_{P}$ and the weak ones with $\sigma_{P}$ for any countable dense set $P \subset \mathbb{R}^{2 N}$, without any assumption on the $m$-bounds or the $l$-bounds, whatsoever. The same result holds when we assume the 
usual Kamke conditions $\left(\mathbf{K}_{x}\right)$ and $\left(\mathbf{K}_{y}\right)$, as well as a very mild hypothesis $\left(\mathbf{L}_{x}\right)$ on the Lipschitz character of the components of the vector field. These are the main conclusions of this section, from which the mentioned continuity results are then derived.

Section 4 provides dynamical methods to study the long-term behavior of the trajectories for each of the global, continuous and monotone skew-product semiflows studied in the previous section. In particular, the notions of semiequilibrium and semicontinuous semiequilibrium introduced in Chuesov [6] and Novo et al. [19] are adapted to this situation, and under adequate conditions, we show that a semicontinuous semiequilibrium guarantees the existence of a semicontinuous equilibrium that determines a closed invariant set of the phase space. When the functions of $E$ are sublinear, $E \times \mathcal{C}^{+}$is positively invariant, and a global, continuous, monotone and sublinear skew-product semiflow $\Phi$ is induced there. In this case, under appropriate assumptions, we prove the existence of invariant subsets $E_{-}, E_{+} \subset E$ such that the restriction of the semiflow to $E_{-} \times \mathcal{C}^{+}$has an unique equilibrium that is continuous, and whose graph is the pullback attractor for the evolution processes. Moreover, this equilibrium is also the forward attractor for the trajectories in $\left(E_{-} \cap E_{+}\right) \times \mathcal{C}^{+}$.

In section 5 the conclusions of the paper are applied to non-autonomous models in mathematical biology. The first example is a model in population dynamics defined by a scalar Carathéodory delay differential equation for which the existence of a maximal and a minimal bounded equilibria is deduced. Under appropriate sublinearity assumptions, the existence of a unique continuous equilibrium whose graph coincides with the pullback attractor for the evolution processes is shown. The second example is the mathematical model of biochemical feedback in protein synthesis given by a system of Carathéodory ordinary differential equations, that has been extensively studied in the literature in other deterministic and random versions. See for example Selgrade [23], Smith [26], Smith and Thieme [27], Krause and Ranft [12], Chuesov [6] and Novo et al. [19].

\section{Preliminary notions And Results}

2.1. Spaces and topologies. We will denote by $\mathbb{R}^{N}$ the $N$-dimensional euclidean space with norm $|\cdot|$ and by $B_{r}$ the closed ball of $\mathbb{R}^{N}$ centered at the origin and with radius $r$. When $N=1$ we will simply write $\mathbb{R}$ and the symbol $\mathbb{R}^{+}$will denote the set of positive real numbers. Unless otherwise noted, $p$ will denote a natural number $1 \leqslant p<\infty$. Moreover, for any interval $I \subseteq \mathbb{R}$ and any $W \subset \mathbb{R}^{N}$, we will use the following notation

$\mathcal{C}(I, W)$ : space of continuous functions from $I$ to $W$ endowed with the norm $\|\cdot\|_{\infty}$. In particular, we will denote by $\mathcal{C}:=\mathcal{C}\left([-1,0], \mathbb{R}^{N}\right)$.

$L^{p}\left(I, \mathbb{R}^{N}\right)$ : space of measurable functions from $I$ to $\mathbb{R}^{N}$ whose norm is in the Lebesgue space $L^{p}(I)$.

$L_{\text {loc }}^{p}\left(\mathbb{R}^{N}\right)$ : space of functions $x: \mathbb{R} \rightarrow \mathbb{R}^{N}$ such that for every compact interval $I \subset \mathbb{R}, x$ belongs to $L^{p}\left(I, \mathbb{R}^{N}\right)$. When $N=1$, we will simply write $L_{l o c}^{p}$.

We will consider, and denote by $\mathfrak{C}_{p}\left(\mathbb{R}^{M}, \mathbb{R}^{N}\right)$ (or simply $\mathfrak{C}_{p}$ when $M=N$ ), the set of functions $f: \mathbb{R} \times \mathbb{R}^{M} \rightarrow \mathbb{R}^{N}$ satisfying

(C1) $f$ is Borel measurable and 
(C2) for every compact set $K \subset \mathbb{R}^{M}$ there exists a real-valued function $m^{K} \in L_{l o c}^{p}$, called $m$-bound in the following, such that for almost every $t \in \mathbb{R}$ one has $|f(t, x)| \leqslant m^{K}(t)$ for any $x \in K$.

Now we introduce the sets of Carathéodory functions which are used in the rest of the work.

Definition 2.1 (Lipschitz Carathéodory functions). A function $f: \mathbb{R} \times \mathbb{R}^{M} \rightarrow \mathbb{R}^{N}$ is said to be Lipschitz Carathéodory, and we will write $f \in \mathfrak{L C}_{p}\left(\mathbb{R}^{M}, \mathbb{R}^{N}\right.$ ) (or simply $f \in \mathfrak{L} \mathfrak{C}_{p}$ when $M=N$ ), if it satisfies (C1), (C2) and

(L) for every compact set $K \subset \mathbb{R}^{M}$ there exists a real-valued function $l^{K} \in L_{\text {loc }}^{p}$ such that for almost every $t \in \mathbb{R}$ one has $\left|f\left(t, x_{1}\right)-f\left(t, x_{2}\right)\right| \leqslant l^{K}(t)\left|x_{1}-x_{2}\right|$ for any $x_{1}, x_{2} \in K$.

In particular, for any compact set $K \subset \mathbb{R}^{M}$, we refer to the optimal $m$-bound and the optimal l-bound of $f$ as to

$$
m^{K}(t)=\sup _{x \in K}|f(t, x)| \quad \text { and } \quad l^{K}(t)=\sup _{\substack{x_{1}, x_{2} \in K \\ x_{1} \neq x_{2}}} \frac{\left|f\left(t, x_{1}\right)-f\left(t, x_{2}\right)\right|}{\left|x_{1}-x_{2}\right|},
$$

respectively. Clearly, for any compact set $K \subset \mathbb{R}^{M}$ the suprema in (2.1) can be taken for a countable dense subset of $K$ leading to the same actual definition, which guarantees that the functions defined in (2.1) are measurable.

Definition 2.2 (Strong Carathéodory functions). A function $f: \mathbb{R} \times \mathbb{R}^{M} \rightarrow \mathbb{R}^{N}$ is said to be strong Carathéodory, and we will write $f \in \mathfrak{S C}_{p}\left(\mathbb{R}^{M}, \mathbb{R}^{N}\right.$ ) (or simply $f \in \mathfrak{S C}_{p}$ when $M=N$ ), if it satisfies (C1), (C2) and

(SC) for almost every $t \in \mathbb{R}$, the function $f(t, \cdot)$ is continuous.

The concept of optimal $m$-bound for a strong Carathéodory function on any compact set $K \subset \mathbb{R}^{N}$, is defined exactly as in equation (2.1).

Remark 2.3. As regards Definitions 2.1 and 2.2, when $p=1$, we will omit the number 1 from the notation. For example, we will simply write $\mathfrak{L} \mathfrak{C}$ instead of $\mathfrak{L C}_{1}$. Moreover, the functions which lay in the same set and only differ on a negligible subset of $\mathbb{R}^{1+M}$ will be identified. Therefore, one automatically has that $\mathfrak{L C}_{p}\left(\mathbb{R}^{M}, \mathbb{R}^{N}\right) \subset \mathfrak{S} \mathfrak{C}_{p}\left(\mathbb{R}^{M}, \mathbb{R}^{N}\right)$.

Definition 2.4 ( $l_{1}$ - and $l_{2}$-bounds). Let us consider a function $f \in \mathfrak{S C}_{p}\left(\mathbb{R}^{2 N}, \mathbb{R}^{N}\right)$. We say that $f$ admits $l_{1}$-bounds (resp. $l_{2}$-bounds) if for every $j \in \mathbb{N}$ there exists a function $l_{1}^{j} \in L_{l o c}^{p}\left(\right.$ resp. $\left.l_{2}^{j} \in L_{l o c}^{p}\right)$ such that for almost every $t \in \mathbb{R}$

$$
\left|f\left(t, x_{1}, y\right)-f\left(t, x_{2}, y\right)\right| \leqslant l_{1}^{j}(t)\left|x_{1}-x_{2}\right| \quad \text { for all }\left(x_{1}, y\right),\left(x_{2}, y\right) \in B_{j}
$$

(resp. $\left|f\left(t, x, y_{1}\right)-f\left(t, x, y_{2}\right)\right| \leqslant l_{2}^{j}(t)\left|y_{1}-y_{2}\right| \quad$ for all $\left.\left(x, y_{1}\right),\left(x, y_{2}\right) \in B_{j}\right)$.

If $f \in \mathfrak{S C}_{p}\left(\mathbb{R}^{2 N}, \mathbb{R}^{N}\right)$ admits $l_{1}$-bounds (resp. $l_{2}$-bounds), for every $j \in \mathbb{N}$ we refer to the optimal $l_{1}$-bound (resp. the optimal $l_{2}$-bound) for $f$ on $B_{j} \subset \mathbb{R}^{2 N}$ as to

$$
\begin{aligned}
l_{1}^{j}(t) & =\sup _{\substack{\left(x_{1}, y\right),\left(x_{2}, y\right) \in B_{j} \\
x_{1} \neq x_{2}}} \frac{\left|f\left(t, x_{1}, y\right)-f\left(t, x_{2}, y\right)\right|}{\left|x_{1}-x_{2}\right|} \\
\left(\operatorname{resp.} l_{2}^{j}(t)\right. & \left.=\sup _{\substack{\left(x, y_{1}\right),\left(x, y_{2}\right) \in B_{j} \\
y_{1} \neq y_{2}}} \frac{\left|f\left(t, x, y_{1}\right)-f\left(t, x, y_{2}\right)\right|}{\left|y_{1}-y_{2}\right|}\right) .
\end{aligned}
$$


Notice also that, if $f \in \mathfrak{L C}_{p}\left(\mathbb{R}^{2 N}, \mathbb{R}^{N}\right)$, then $f$ always admits both $l_{1}$-bounds and $l_{2}$-bounds, and it is easy to prove that for all $t \in \mathbb{R}$ one has $l^{j}(t) \leqslant l_{1}^{j}(t)+l_{2}^{j}(t)$, where by $l^{j}$ we denote the optimal $l$-bound for $f$ on $B_{j}$ as in (2.1).

We endow the space $\mathfrak{S C}\left(\mathbb{R}^{M}, \mathbb{R}^{N}\right)$ with strong and weak metric topologies which rely on the notion of suitable set of moduli of continuity as proposed in [13] and [14]. We, firstly recall such definition.

Definition 2.5 (Suitable set of moduli of continuity). We call a suitable set of moduli of continuity, any countable set of non-decreasing continuous functions

$$
\Theta=\left\{\theta_{j}^{I} \in C\left(\mathbb{R}^{+}, \mathbb{R}^{+}\right) \mid j \in \mathbb{N}, I=\left[q_{1}, q_{2}\right], q_{1}, q_{2} \in \mathbb{Q}\right\}
$$

such that $\theta_{j}^{I}(0)=0$ for every $\theta_{j}^{I} \in \Theta$, and with the relation of partial order given by

$$
\theta_{j_{1}}^{I_{1}} \leqslant \theta_{j_{2}}^{I_{2}} \quad \text { whenever } I_{1} \subseteq I_{2} \text { and } j_{1} \leqslant j_{2} .
$$

As follows, we recall some of the hybrid topologies on $\mathfrak{S C}\left(\mathbb{R}^{N+M}, \mathbb{R}^{N}\right)$ introduced in [15]. Notice that, as a rule, when inducing a topology on a subspace we will denote the induced topology with the same symbol used for the topology on the original space.

Definition 2.6 (Hybrid topologies on $\mathfrak{S C}\left(\mathbb{R}^{N+M}, \mathbb{R}^{N}\right)$ ). Let $\Theta$ and $\widehat{\Theta}$ be suitable sets of moduli of continuity as in Definition 2.5, $D$ be a countable dense subset of $\mathbb{R}^{M}$ and, for any $I=\left[q_{1}, q_{2}\right], q_{1}, q_{2} \in \mathbb{Q}$ and $j \in \mathbb{N}$, let $\mathcal{K}_{j}^{I}$ and $\hat{\mathcal{K}}_{j}^{I}$ be the sets of functions in $C\left(I, \mathbb{R}^{N}\right)$ whose modulus is bounded by $j$ and which admit $\theta_{j}^{I}$ and $\hat{\theta}_{j}^{I}$, respectively, as a moduli of continuity.

- $\mathcal{T}_{\Theta D}\left(\right.$ resp. $\left.\sigma_{\Theta D}\right)$ is the topology on $\mathfrak{S C}_{p}\left(\mathbb{R}^{N+M}, \mathbb{R}^{N}\right)\left(\operatorname{resp} . \mathfrak{S} \mathfrak{C}\left(\mathbb{R}^{N+M}, \mathbb{R}^{N}\right)\right)$ generated by the family of seminorms

$$
\begin{gathered}
p_{I, y, j}(f)=\sup _{x \in \mathcal{K}_{j}^{I}}\left[\int_{I}|f(t, x(t), y)|^{p} d t\right]^{1 / p}, \\
\left(\text { resp. } p_{I, y, j}(f)=\sup _{x \in \mathcal{K}_{j}^{I}}\left|\int_{I} f(t, x(t), y) d t\right|\right)
\end{gathered}
$$

with $f \in \mathfrak{S C}_{p}\left(\mathbb{R}^{N+M}, \mathbb{R}^{N}\right)$ (resp. $\left.f \in \mathfrak{S C}\left(\mathbb{R}^{N+M}, \mathbb{R}^{N}\right)\right), I=\left[q_{1}, q_{2}\right], q_{1}, q_{2} \in \mathbb{Q}$, $y \in D$ and $j \in \mathbb{N}$. Both $\left(\mathfrak{S C}_{p}\left(\mathbb{R}^{N+M}, \mathbb{R}^{N}\right), \mathcal{T}_{\Theta D}\right)$ and $\left(\mathfrak{S C}\left(\mathbb{R}^{N+M}, \mathbb{R}^{N}\right), \sigma_{\Theta D}\right)$ are locally convex metric spaces.

- $\mathcal{T}_{\Theta \Theta}\left(\right.$ resp. $\left.\sigma_{\Theta} \hat{\Theta}\right)$ is the topology on $\mathfrak{S C}_{p}\left(\mathbb{R}^{N+M}, \mathbb{R}^{N}\right)\left(\right.$ resp. $\left.\mathfrak{S C}\left(\mathbb{R}^{N+M}, \mathbb{R}^{N}\right)\right)$ generated by the family of seminorms

$$
\begin{gathered}
p_{I, j}(f)=\sup _{x \in \mathcal{K}_{j}^{I}, y \in \hat{\mathcal{K}}_{j}^{I-1}}\left[\int_{I}|f(t, x(t), y(t-1))|^{p} d t\right]^{1 / p}, \\
\left(\text { resp. } p_{I, j}(f)=\sup _{x \in \mathcal{K}_{j}^{I}, y \in \hat{\mathcal{K}}_{j}^{I-1}}\left|\int_{I} f(t, x(t), y(t-1)) d t\right|\right)
\end{gathered}
$$

with $f \in \mathfrak{S C}_{p}\left(\mathbb{R}^{N+M}, \mathbb{R}^{N}\right)\left(\right.$ resp. $\left.f \in \mathfrak{S} \mathfrak{C}\left(\mathbb{R}^{N+M}, \mathbb{R}^{N}\right)\right), I=\left[q_{1}, q_{2}\right], q_{1}, q_{2} \in \mathbb{Q}$ and $j \in \mathbb{N}$. One has that $\left(\mathfrak{S C}_{p}\left(\mathbb{R}^{N+M}, \mathbb{R}^{N}\right), \mathcal{T}_{\Theta} \hat{\Theta}\right)$ and $\left(\mathfrak{S C}\left(\mathbb{R}^{N+M}, \mathbb{R}^{N}\right), \sigma_{\Theta} \hat{\Theta}\right)$ are locally convex metric spaces 
Remark 2.7. The hybrid topologies $\mathcal{T}_{\Theta} \hat{\Theta}$ and $\sigma_{\Theta} \hat{\Theta}$ can be equivalently constructed using families of seminorms for which $y \in \widehat{\mathcal{K}}_{j}^{I}$ instead of $\widehat{\mathcal{K}}_{j}^{I-1}$ (and of course $y$ is evaluated at $t$ instead of $t-1$ ). For further details, see [15, Lemma 2.12]. We will use this fact consistently in the following. Moreover, notice that when $M=0$, all the previous topologies reduce either to $\mathcal{T}_{\Theta}$ or $\sigma_{\Theta}$, that is, the topologies generated by the families of seminorms

$$
p_{I, j}(f)=\sup _{x \in \mathcal{K}_{j}^{I}}\left[\int_{I}|f(t, x(t))|^{p} d t\right]^{1 / p}\left(\operatorname{resp} \cdot p_{I, j}(f)=\sup _{x \in \mathcal{K}_{j}^{I}}\left|\int_{I} f(t, x(t)) d t\right|\right)
$$

with $f \in \mathfrak{S C}_{p}\left(\mathbb{R}^{N}, \mathbb{R}^{N}\right)\left(\right.$ resp. $\left.f \in \mathfrak{S C}\left(\mathbb{R}^{N}, \mathbb{R}^{N}\right)\right), I=\left[q_{1}, q_{2}\right], q_{1}, q_{2} \in \mathbb{Q}$, and $j \in \mathbb{N}$.

Remark 2.8. From this point on, we will only consider the case $p=1$. The reason is twofold. On one side, the case $p>1$ only applies to strong topologies, and the arguments employed in the proofs for $p=1$ can be extended with small modifications also when $p>1$; all the presented results still hold true. On the other hand, this choice allows to shorten the statement of most of the results because the case of strong and weak topologies can be presented simultaneously.

We state the following technical lemma for the weak hybrid topologies, which will be useful in the following. We skip the proof because it differs only in minor details from the one of Lemma 2.13 in [14].

Lemma 2.9. Let $D$ be a dense and countable subset of $\mathbb{R}^{M}$ and $\Theta, \widehat{\Theta}$ be any pair of suitable sets of moduli of continuity. Moreover, for each $j \in \mathbb{N}$ and $I=\left[q_{1}, q_{2}\right]$, $q_{1}, q_{2} \in \mathbb{Q}$, let $\mathcal{K}_{j}^{I}$ and $\widehat{\mathcal{K}}_{j}^{I}$ be the compact sets in $C\left(I, B_{j}\right)$ which admit $\theta_{j}^{I} \in \Theta$ and $\hat{\theta}_{j}^{I} \in \widehat{\Theta}$, respectively, as moduli of continuity.

(i) Consider $f \in \mathfrak{S C}\left(\mathbb{R}^{N+M}, \mathbb{R}^{N}\right)$. If $\left(x_{n}\right)_{n \in \mathbb{N}}$ is a sequence in $\mathcal{K}_{j}^{I}$ converging uniformly to some function $x \in \mathcal{K}_{j}^{I}$ and $\left(y_{n}\right)_{n \in \mathbb{N}}$ is a sequence in $\widehat{\mathcal{K}}_{j}^{I-1}$ converging uniformly to some function $y \in \widehat{\mathcal{K}}_{j}^{I-1}$. Then

$$
\lim _{n \rightarrow \infty} \int_{p_{1}}^{p_{2}} f\left(t, x_{n}(t), y_{n}(t-1)\right) d t=\int_{p_{1}}^{p_{2}} f(t, x(t), y(t-1)) d t,
$$

whenever $p_{1}, p_{2} \in \mathbb{Q}$ and $q_{1} \leqslant p_{1}<p_{2} \leqslant q_{2}$.

(ii) Let $\left(g_{n}\right)_{n \in \mathbb{N}}$ be a sequence in $\mathfrak{S C}\left(\mathbb{R}^{N+M}, \mathbb{R}^{N}\right)$ converging to some function $g$ in $\left(\mathfrak{S C}\left(\mathbb{R}^{N}\right), \sigma_{\Theta} \hat{\Theta}\right)$ (resp. in $\left.\left(\mathfrak{S C}\left(\mathbb{R}^{N}\right), \sigma_{\Theta D}\right)\right)$. Then,

$$
\begin{aligned}
& \lim _{n \rightarrow \infty} \sup _{x \in \mathcal{K}_{j}^{I}, y \in \hat{\mathcal{K}}_{j}^{I-1}}\left|\int_{p_{1}}^{p_{2}}\left[g_{n}(t, x(t), y(t-1))-g(t, x(t), y(t-1))\right] d t\right|=0, \\
& \left(\text { resp. for any } y \in D, \quad \lim _{n \rightarrow \infty} \sup _{x \in \mathcal{K}_{j}^{I}}\left|\int_{p_{1}}^{p_{2}}\left[g_{n}(t, x(t), y)-g(t, x(t), y)\right] d t\right|=0\right) \\
& \text { whenever } p_{1}, p_{2} \in \mathbb{Q} \text { and } q_{1} \leqslant p_{1}<p_{2} \leqslant q_{2} .
\end{aligned}
$$

We conclude this subsection by recalling the notion of $L_{l o c}^{1}$-equicontinuity and proving some results on the previously outlined topological spaces when such property holds. 
Definition 2.10 ( $L_{l o c}^{1}$-equicontinuity). A set $S$ of positive functions in $L_{l o c}^{1}$ is $L_{l o c}^{1}$ equicontinuous if for any $r>0$ and for any $\varepsilon>0$ there exists a $\delta=\delta(r, \varepsilon)>0$ such that, for any $-r \leqslant s \leqslant t \leqslant r$, with $t-s<\delta$, the following inequality holds

$$
\sup _{m \in S} \int_{s}^{t} m(u) d u<\varepsilon \text {. }
$$

The following definition extends the previous notion to sets of Carathéodory functions through their $m$-bounds.

Definition 2.11 ( $L_{l o c}^{1}$-equicontinuous $m$-bounds). We say that

(i) a set $E \subset \mathfrak{C}\left(\mathbb{R}^{M}, \mathbb{R}^{N}\right)$ has $L_{\text {loc }}^{1}$-equicontinuous $m$-bounds, if for any $j \in \mathbb{N}$ there exists a set $S^{j} \subset L_{l o c}^{1}$ of $m$-bounds of the functions of $E$ on $B_{j}$, such that $S^{j}$ is $L_{l o c}^{1}$-equicontinuous;

(ii) $f \in \mathfrak{C}\left(\mathbb{R}^{M}, \mathbb{R}^{N}\right)$ has $L_{\text {loc }}^{1}$-equicontinuous $m$-bounds if the set $\left\{f_{t} \mid t \in \mathbb{R}\right\}$ admits $L_{l o c}^{1}$-equicontinuous $m$-bounds.

For the sake of completeness, we also include the following statement which covers a particular case of Proposition 2.19 in [15]. Such result guarantees that the property of $L_{l o c}^{1}$-equicontinuity is satisfied also in the closure in $\mathfrak{S C}_{p}\left(\mathbb{R}^{2 N}, \mathbb{R}^{N}\right)$ with respect of the topologies $\mathcal{T}_{\Theta D}$ and $\sigma_{\Theta D}$ (and thus also with respect to any topology of the type $\mathcal{T}_{\Theta} \hat{\Theta}$ and $\left.\sigma_{\Theta} \hat{\Theta}\right)$. In fact, the proof (that can be found in [15]) shows that such result is also qualitative in the sense that the limit functions satisfy the considered inequalities with respect to the same constants.

Proposition 2.12. Let $E$ be a subset of $\mathfrak{S C}\left(\mathbb{R}^{M}, \mathbb{R}^{N}\right)$ with $L_{\text {loc }}^{1}$-equicontinuous $m$-bounds. Then, the closure of $E$ in $\left(\mathfrak{S C}\left(\mathbb{R}^{M}, \mathbb{R}^{N}\right), \mathcal{T}\right)$, i.e. $\operatorname{cls}_{\left(\mathfrak{S C}\left(\mathbb{R}^{M}, \mathbb{R}^{N}\right), \mathcal{T}\right)}(E)$, has $L_{\text {loc }}^{1}$-equicontinuous m-bounds, where $\mathcal{T}$ is $\mathcal{T}_{\Theta D}$ or $\sigma_{\Theta D}$.

Remark 2.13. If $E \subset \mathfrak{L C}\left(\mathbb{R}^{2 N}, \mathbb{R}^{N}\right)$ has $L_{\text {loc }}^{1}$-equicontinuous $m$-bounds, then one can define a specific suitable set of moduli of continuity as follows: for any interval $I=\left[q_{1}, q_{2}\right], q_{1}, q_{2} \in \mathbb{Q}$, define

$$
\theta_{j}^{I}(s):=\sup _{t \in I, f \in E} \int_{t}^{t+s} m_{f}^{j}(u) d u,
$$

where, for any $f \in E$, the function $m_{f}^{j} \in L_{l o c}^{1}$ denotes the optimal $m$-bounds of $f$ on $B_{j}$. In particular, if we deal with just one function $f \in \mathfrak{L} \mathfrak{C}\left(\mathbb{R}^{2 N}, \mathbb{R}^{N}\right)$ with $L_{\text {loc }}^{1}$-equicontinuous $m$-bounds, one can consider the set $E=\left\{f_{t} \mid t \in \mathbb{R}\right\}$ of the time translations of $f$ and still define a suitable set of moduli of continuity as before. Notice, however, that in this case the elements of $\Theta$ are independent of the interval $I$, that is, for any $j \in \mathbb{N}$ and $I=\left[q_{1}, q_{2}\right], q_{1}, q_{2} \in \mathbb{Q}$ one has that $\theta_{j}^{I}$ in (2.2), coincides in fact with $\theta_{j}(s):=\sup _{t \in \mathbb{R}} \int_{t}^{t+s} m^{j}(u) d u$, where $m^{j}$ is the optimal $m$-bound for $f$ on $B_{j}$. In either case, we will say that $\Theta$ is the suitable set of moduli of continuity given by the $m$-bounds of, respectively, $E$ or $f$.

2.2. Carathéodory delay differential equations. For the sake of completeness and to set some notation, we include the statement of a theorem of existence, uniqueness and continuous variation of the solution for a Cauchy Problem of Carathéodory type with constant delay. A proof can be derived by the one given for Carathéodory ordinary differential equations in Coddington and Levinson [7, Theorems $1.1,1.2,2.2,4.2$, and 4.3]. We recall that $\mathcal{C}$ denotes the set $\mathcal{C}\left([-1,0], \mathbb{R}^{N}\right)$. 
Theorem 2.14. For any $f \in \mathfrak{L C}\left(\mathbb{R}^{2 N}, \mathbb{R}^{N}\right)$ and any $\phi \in \mathcal{C}$ there exists a maximal interval $I_{f, \phi}=\left[-1, b_{f, \phi}\right)$ and a unique continuous function $x(\cdot, f, \phi)$ defined on $I_{f, \phi}$ which is the solution of the delay differential problem

$$
\begin{cases}x^{\prime}(t)=f(t, x(t), x(t-1)) & \text { for } t>0 \\ x(t)=\phi(t) & \text { for } t \in[-1,0] .\end{cases}
$$

In particular, if $b_{f, \phi}<\infty$, then $|x(t, f, \phi)| \rightarrow \infty$ as $t \rightarrow b_{f, \phi}$. Moreover, for any compact interval $I=[-1, b] \subset I_{f, \phi}$ and any $\varepsilon>0$, there exists $\delta=\delta(I, \varepsilon)>0$ such that for any $\psi \in \mathcal{C}$, if $\|\phi-\psi\|_{\mathcal{C}}<\delta$, then $x(\cdot, f, \psi)$ exists on $[-1, b]$, and $\|x(\cdot, f, \phi)-x(\cdot, f, \psi)\|_{\mathcal{C}([-1, b])}<\varepsilon$.

As usual, for each $t>0$ belonging to $I_{f, \phi}$ we will denote by $x_{t}(\cdot, f, \phi)$ the function of $\mathcal{C}$ defined as

$$
x_{t}(s, f, \phi)=x(s+t, f, \phi) \quad \text { whenever } s \in[-1,0] .
$$

For each $s \in \mathbb{R}$ the function $f_{s} \in \mathfrak{L} \mathfrak{C}\left(\mathbb{R}^{2 N}, \mathbb{R}^{N}\right)$ denotes the time-translation of $f$

$$
f_{s}: \mathbb{R} \times \mathbb{R}^{2 N} \rightarrow \mathbb{R}^{N}, \quad(t, x) \mapsto f_{s}(t, x)=f(s+t, x) .
$$

A delay differential equation like (2.3) induces a skew-product semiflow

$$
\mathcal{U} \subset \mathbb{R}^{+} \times\left\{f_{s} \mid s \in \mathbb{R}\right\} \times \mathcal{C} \rightarrow\left\{f_{s} \mid s \in \mathbb{R}\right\} \times \mathcal{C}, \quad(t, g, \phi) \mapsto\left(g_{t}, x_{t}(\cdot, g, \phi)\right) .
$$

Instead of just one differential equation, one can consider a whole set of problems whose vector fields belong to a set $E \subset \mathfrak{L} \mathfrak{C}\left(\mathbb{R}^{2 N}, \mathbb{R}^{N}\right)$. In order to construct a skewproduct semiflow from $E$, one has to consider all the possible time-translations of any element in it (the same way we passed from $f$ to $\left\{f_{s} \mid s \in \mathbb{R}\right\}$ ). Instead of burdening the notation, we will then require $E$ to be invariant with respect to the function

$$
\mathbb{R} \times \mathfrak{L} \mathfrak{C}\left(\mathbb{R}^{2 N}, \mathbb{R}^{N}\right) \rightarrow \mathfrak{L} \mathfrak{C}\left(\mathbb{R}^{2 N}, \mathbb{R}^{N}\right), \quad(s, f) \mapsto f_{s},
$$

that we will call base flow in the following. Then, the skew-product semiflow induced by $E$ is

$$
\mathcal{U} \subset \mathbb{R}^{+} \times E \times \mathcal{C} \rightarrow E \times \mathcal{C}, \quad(t, f, \phi) \mapsto\left(f_{t}, x_{t}(\cdot, f, \phi)\right) .
$$

The aim of this paper is to provide conditions and optimal topologies on invariant closed subsets $E \subset \mathfrak{L} \mathfrak{C}\left(\mathbb{R}^{2 N}, \mathbb{R}^{N}\right)$ with suitable monotonicity requisites, so that (2.4) is continuous. Specifically, in this work no assumptions on the $l$-bounds of $E$ are made.

As a matter of fact, our first result is to show that the function in (2.4) can still be made continuous if restricted to a smaller subset of the phase-space (although in such a way it ceases to be a skew-product semiflow). Let $\Theta$ be a suitable set of moduli of continuity as in Definition 2.5 , and $\theta_{0} \in C\left(\mathbb{R}^{+}, \mathbb{R}^{+}\right)$be a further modulus of continuity. Consider the set

$$
\mathcal{F}_{0}:=\left\{\phi \in \mathcal{C} \mid \bmod (\phi) \leqslant \theta_{0}\right\},
$$

and the suitable set of moduli of continuity

$$
\bar{\Theta}=\left\{\bar{\theta}_{j}^{I} \in C\left(\mathbb{R}^{+}, \mathbb{R}^{+}\right) \mid \bar{\theta}_{j}^{I}(s)=\max \left\{\theta_{j}^{I}(s), \theta_{0}(s)\right\}\right\},
$$

and let $\mathcal{T}_{\Theta} \bar{\Theta}$ and $\sigma_{\Theta} \bar{\Theta}$ be the topologies constructed from $\Theta$ and $\bar{\Theta}$ as in Definition 2.6. If $E$ is any subset of $\mathfrak{L C}\left(\mathbb{R}^{2 N}, \mathbb{R}^{N}\right)$, let us denote by $\bar{E}_{\mathcal{T}_{\ominus \bar{\Theta}}}$ and by $\bar{E}_{\sigma_{\Theta \bar{\Theta}}}$ 
the closure of $E$ in $\left(\mathfrak{L C}\left(\mathbb{R}^{2 N}, \mathbb{R}^{N}\right), \mathcal{T}_{\Theta \bar{\Theta}}\right)$ and in $\left(\mathfrak{L} \mathfrak{C}\left(\mathbb{R}^{2 N}, \mathbb{R}^{N}\right), \sigma_{\Theta} \bar{\Theta}\right)$, respectively, and let $\mathcal{U}_{\mathcal{T}_{\ominus \bar{\Theta}}} \subset \mathbb{R}^{+} \times \bar{E}_{\mathcal{T}_{\ominus \bar{\Theta}}} \times \mathcal{F}_{0}$ and $\mathcal{U}_{\sigma_{\Theta \bar{\Theta}}} \subset \mathbb{R}^{+} \times \bar{E}_{\sigma_{\Theta \bar{\Theta}}} \times \mathcal{F}_{0}$ be defined by

$$
\mathcal{U}_{\mathcal{T}_{\Theta \widehat{\Theta}}}=\bigcup_{\substack{f \in \bar{E}_{\mathcal{T}_{\Theta \widehat{\Theta}}}, \phi \in \mathcal{F}_{0}}}\left\{(t, f, \phi) \mid t \in I_{f, \phi}\right\} \quad \text { and } \quad \mathcal{U}_{\sigma_{\Theta \widehat{\Theta}}}=\bigcup_{\substack{f \in \bar{E}_{\sigma_{\Theta \widehat{\Theta}}} \\ \phi \in \mathcal{F}_{0}}}\left\{(t, f, \phi) \mid t \in I_{f, \phi}\right\} .
$$

Theorem 2.15. Consider $E \subset \mathfrak{L C}\left(\mathbb{R}^{2 N}, \mathbb{R}^{N}\right)$ with $L_{\text {loc }}^{1}$-equicontinuous $m$-bounds and let $\Theta$ be the suitable set of moduli of continuity given by the m-bounds in Remark 2.13.

(i) Let $\left(\phi_{n}\right)_{n \in \mathbb{N}}$ be a sequence converging uniformly to $\phi$ in $\mathcal{C}$ and let $\theta_{0}$ be the modulus of continuity shared by the functions $\left\{\phi_{n} \mid n \in \mathbb{N}\right\} \cup\{\phi\}$. Furthermore, let $\bar{\Theta}$ be the suitable set of moduli of continuity constructed in (2.6). If $\left(f_{n}\right)_{n \in \mathbb{N}}$ is a sequence in $E$ converging to $f$ in $\left(\mathfrak{L C}\left(\mathbb{R}^{2 N}, \mathbb{R}^{N}\right), \sigma_{\Theta} \bar{\Theta}\right)$, then, with the notation of Theorem 2.3, one has that

$$
x\left(\cdot, f_{n}, \phi_{n}\right) \stackrel{n \rightarrow \infty}{\longrightarrow} x(\cdot, f, \phi)
$$

uniformly in any $[-1, T] \subset I_{f, \phi}$.

(ii) If additionally $E$ is invariant with respect to the base flow, $\bar{E}$ denotes the closure of $E$ in $\left(\mathfrak{L} \mathfrak{C}\left(\mathbb{R}^{2 N}, \mathbb{R}^{N}\right), \mathcal{T}\right)$, where $\mathcal{T} \in\left\{\mathcal{T}_{\Theta} \bar{\Theta}, \sigma_{\Theta} \bar{\Theta}\right\}$, and $\mathcal{F}_{0}$ is the set constructed as in (2.5) then the map

$$
\Phi: \mathcal{U}_{\mathcal{T}} \subset \mathbb{R}^{+} \times \bar{E} \times \mathcal{F}_{0} \rightarrow \bar{E} \times \mathcal{C}, \quad(t, f, \phi) \mapsto\left(f_{t}, x_{t}(\cdot, f, \phi)\right)
$$

is continuous.

Proof. The proof of (i) is given in [15, Theorem 3.6]. On the other hand, (ii) is a consequence of (i) and of the continuity of the function $(t, f) \mapsto f_{t}$ proved in $[15$, Theorem 3.8].

\section{Monotone SEmiflows}

In this section, we address the problem of the global continuity for the skewproduct semiflows induced by Carathéodory delay differential equations whose vector fields satisfy a specific property of monotonicity. We initially recall the definition of Kamke's conditions. In this setup, they split into two properties of monotonicity, $\left(\mathbf{K}_{x}\right)$ and $\left(\mathbf{K}_{y}\right)$, one for each spatial variable. Then, we prove several technical results. For example, we show that $\left(\mathbf{K}_{x}\right)$ and $\left(\mathbf{K}_{y}\right)$ are robust with respect to the limits in the hybrid topologies, and that $\left(\mathbf{K}_{y}\right)$ is sufficient to provide equivalence of the hybrid topologies (either weak or strong). As a consequence, we obtain that the skew-product semiflow induced by a set of Carathéodory functions satisfying $\left(\mathbf{K}_{y}\right)$, is continuous if endowed with $\mathcal{T}_{\Theta D}$ or $\sigma_{\Theta D}$. Finally, we recall the definition of monotone skew-product semiflows and prove that $\left(\mathbf{K}_{x}\right)$ and $\left(\mathbf{K}_{y}\right)$ together, characterize a monotone skew-product semiflow. Hence, we obtain the continuity of such semiflows as a corollary of the previous results. The last part of the section deals with a stronger assumption of monotonicity which allows to obtain two additional results of continuity of the skew-product semiflow when pointwise (and thus weaker) topologies are used. Moreover, we relate the previous results to Carathéodory ordinary differential equations. 
From the usual componentwise strong partial ordering in $\mathbb{R}^{N}$, that is,

$$
\begin{array}{lll}
y \leqslant z & \Longleftrightarrow \quad y_{i} \leqslant z_{i} & \text { for } i=1, \ldots, N \\
y<z & \Longleftrightarrow \quad y \leqslant z \quad \text { and } y_{i}<z_{i} \text { for some } i \in\{1, \ldots, N\}, \\
y \ll z & \Longleftrightarrow \quad y_{i}<z_{i} & \text { for } i=1, \ldots, N
\end{array}
$$

we obtain a strong partial ordering on $\mathcal{C}=\mathcal{C}\left([-1,0], \mathbb{R}^{N}\right)$ defined by

$$
\begin{aligned}
& \phi \leqslant \psi \quad \Longleftrightarrow \phi(s) \leqslant \psi(s) \text { for each } s \in[-1,0], \\
& \phi<\psi \quad \Longleftrightarrow \phi \leqslant \psi \text { and } \phi \neq \psi, \\
& \phi \ll \psi \quad \Longleftrightarrow \phi(s) \ll \psi(s) \text { for each } s \in[-1,0] .
\end{aligned}
$$

The positive cone is $\mathcal{C}^{+}:=C\left([-1,0],\left(\mathbb{R}^{+}\right)^{N}\right)=\{\phi \in \mathcal{C} \mid \phi \geqslant 0\}$ with nonempty interior $\operatorname{Int} \mathcal{C}^{+}=\{\phi \in \mathcal{C} \mid \phi \gg 0\}$.

Definition 3.1 (Kamke's conditions). We say that $f \in \mathfrak{L} \mathfrak{C}\left(\mathbb{R}^{2 N}, \mathbb{R}^{N}\right)$ satisfies $\left(\mathbf{K}_{x}\right)$ if for any $a, b, c \in \mathbb{R}^{N}$ with $a \leqslant b$ and $a_{k}=b_{k}$ for some $k \in\{1, \ldots, N\}$, then

$$
f_{k}(t, a, c) \leqslant f_{k}(t, b, c), \quad \text { for a.e. } t \in \mathbb{R} ;
$$

$\left(\mathbf{K}_{y}\right)$ if for any $a, b, c \in \mathbb{R}^{N}$ with $b \leqslant c$, then

$$
f_{k}(t, a, b) \leqslant f_{k}(t, a, c), \quad \text { for all } k=1, \ldots, N, \text { and a.e. } t \in \mathbb{R} .
$$

If both $\left(K_{x}\right)$ and $\left(K_{y}\right)$ hold true, we say that $f$ satisfies the Kamke's conditions or, equivalently, that $f$ is cooperative.

Our first result is to show that $\left(\mathbf{K}_{x}\right)$ and $\left(\mathbf{K}_{y}\right)$ can be propagated through the limits in any of the hybrid topologies considered in Definition 2.6.

Proposition 3.2. Consider $E \subset \mathfrak{L} \mathfrak{C}\left(\mathbb{R}^{2 N}, \mathbb{R}^{N}\right)$. Let $\Theta$ be any suitable set of moduli of continuity as in Definition 2.5, $D$ a countable dense subset of $\mathbb{R}^{N}$, and $\bar{E}$ the closure of $E$ in $\left(\mathfrak{L} \mathfrak{C}\left(\mathbb{R}^{2 N}, \mathbb{R}^{N}\right), \sigma_{\Theta D}\right)$. Then

(i) if any function in $E$ satisfies $\left(\mathbf{K}_{y}\right)$, then also any function in $\bar{E}$ does;

(ii) if any function in $E$ satisfies $\left(\mathbf{K}_{x}\right)$, then also any function in $\bar{E}$ does.

Proof. (i) Consider $f \in \bar{E}$ and a sequence $\left(f_{n}\right)_{n \in \mathbb{N}}$ of functions in $E$ converging to $f$ with respect to $\sigma_{\Theta D}$. First we take $a \in \mathbb{R}^{N}$ and we assume that $b$ and $c$ belongs to $D$. Then, from the convergence in $\sigma_{\Theta D}$ (see Definition 2.6), fixed any compact interval $I \subset \mathbb{R}$ with rational extrema we deduce that

$$
\lim _{n \rightarrow \infty} \int_{I} f_{n}(s, a, b)=\int_{I} f(s, a, b) d s \text { and } \lim _{n \rightarrow \infty} \int_{I} f_{n}(s, a, c)=\int_{I} f(s, a, c) d s .
$$

Moreover, considering $b \leqslant c, k=1, \ldots, N$ and any $t, h \in \mathbb{Q}$ with $h \geqslant 0$, from $\left(\mathbf{K}_{y}\right)$ we obtain

$$
\frac{1}{h} \int_{t}^{t+h}\left(f_{n}\right)_{k}(s, a, b) d s \leqslant \frac{1}{h} \int_{t}^{t+h}\left(f_{n}\right)_{k}(s, a, c) d s
$$

which thanks to $(3.2)$, as $n \rightarrow \infty$ provides

$$
\frac{1}{h} \int_{t}^{t+h} f_{k}(s, a, b) d s \leqslant \frac{1}{h} \int_{t}^{t+h} f_{k}(s, a, c) d s .
$$


Due to the continuity of the integral operator, the previous inequality is satisfied for any $t, h \in \mathbb{R}$, with $h \geqslant 0$. Therefore, using Lebesgue's theorem as $h \rightarrow 0$ we conclude that

$$
f_{k}(t, a, b) \leqslant f_{k}(t, a, c), \quad \text { for a.e. } t \in \mathbb{R},
$$

provided that $b, c \in D$ and $b \leqslant c$. Finally, from $(\mathbf{L})$ and the density of $D$ we deduce the result for any $b, c \in \mathbb{R}^{N}$ which concludes the proof of (i). The proof of (ii) is omitted because it is similar.

Next, we prove a technical lemma which allows us to pass from $\left(\mathbf{K}_{y}\right)$ and $\left(\mathbf{K}_{x}\right)$ (which are formulated pointwise) to a condition of monotonicity which involves continuous functions.

Lemma 3.3. Consider $f \in \mathfrak{L} \mathfrak{C}\left(\mathbb{R}^{2 N}, \mathbb{R}^{N}\right)$. Then

(i) if $f$ satisfies $\left(\mathbf{K}_{y}\right)$, for any interval $I \subset \mathbb{R}$ and any functions $x, y, z \in \mathcal{C}\left(I, \mathbb{R}^{N}\right)$ such that $y(t) \leqslant z(t)$ for all $t \in I$, one has that

$$
f(t, x(t), y(t)) \leqslant f(t, x(t), z(t)), \quad \text { for a.e. } t \in I
$$

(ii) if $f$ satisfies $\left(\mathbf{K}_{x}\right)$, for any interval $I \subset \mathbb{R}$ and any functions $x, y, z \in \mathcal{C}\left(I, \mathbb{R}^{N}\right)$ such that $x(t) \leqslant y(t)$ and $x_{k}(t)=y_{k}(t)$ for all $t \in I$ and some $k \in 1, \ldots, N$, one has that

$$
f_{k}(t, x(t), z(t)) \leqslant f_{k}(t, y(t), z(t)), \quad \text { for a.e. } t \in I .
$$

Proof. Let $D=\left\{s_{n} \mid n \in \mathbb{N}\right\}$ be a dense subset of $I$. From $\left(\mathbf{K}_{y}\right)$ we know that given $n \in \mathbb{N}$ there is a subset $J_{n} \subset I$ of full measure such that

$$
f\left(t, x\left(s_{n}\right), y\left(s_{n}\right)\right) \leqslant f\left(t, x\left(s_{n}\right), z\left(s_{n}\right)\right) \quad \text { for each } t \in J_{n} .
$$

Next we consider the subset $J=\bigcap_{n=1}^{\infty} J_{n} \subset I$, also of full measure and fix $t \in J$. From the density of $D$ we find a subsequence $\left(s_{n_{k}}\right)_{k \in N}$ such that $\lim _{k \rightarrow \infty} s_{n_{k}}=t$, and from (3.4) we deduce that

$$
f\left(t, x\left(s_{n_{k}}\right), y\left(s_{n_{k}}\right)\right) \leqslant f\left(t, x\left(s_{n_{k}}\right), z\left(s_{n_{k}}\right)\right) \quad \text { for each } k \in \mathbb{N} .
$$

Moreover, from $(\mathbf{L})$ and the continuity of the functions $x, y$ and $z$ we obtain

$$
\begin{aligned}
& \lim _{k \rightarrow \infty} f\left(t, x\left(s_{n_{k}}\right), y\left(s_{n_{k}}\right)=f(t, x(t), y(t)),\right. \\
& \lim _{k \rightarrow \infty} f\left(t, x\left(s_{n_{k}}\right), z\left(s_{n_{k}}\right)=f(t, x(t), z(t)),\right.
\end{aligned}
$$

which together with (3.5) yields (3.3) for $t \in J$, and finishes the proof of (i). The proof of (ii) is omitted because it is similar.

The previous results allow to obtain a crucial outcome. All the considered weak (resp. strong) topologies coincide on closed subsets of $\mathfrak{L} \mathfrak{C}\left(\mathbb{R}^{2 N}, \mathbb{R}^{N}\right)$ where all the elements satisfy the property $\left(\mathbf{K}_{y}\right)$.

Proposition 3.4. Let $E \subset \mathfrak{L} \mathfrak{C}\left(\mathbb{R}^{2 N}, \mathbb{R}^{N}\right), \Theta$ and $\widehat{\Theta}$ be any pair of suitable sets of moduli of continuity as in Definition 2.5, and $D$ a countable dense subset of $\mathbb{R}^{N}$. If all the functions in $E$ satisfy $\left(\mathbf{K}_{y}\right)$, then

$$
\operatorname{cls}_{\left(\mathfrak{L} \mathfrak{C}, \sigma_{\Theta} \widehat{\Theta}\right)}(E)=\operatorname{cls}_{\left(\mathfrak{L} \mathfrak{C}, \sigma_{\Theta D}\right)}(E) \quad \text { and } \quad \operatorname{cls}_{\left(\mathfrak{L} \mathfrak{C}, \mathcal{T}_{\Theta} \widehat{\Theta}\right)}(E)=\operatorname{cls}_{\left(\mathfrak{L} \mathfrak{C}, \mathcal{T}_{\Theta D}\right)}(E)
$$


Proof. Firstly, notice that for any pair $\Theta$ and $\widehat{\Theta}$ of suitable sets of moduli of continuity and any countable dense subset $D \subset \mathbb{R}^{N}, \sigma_{\Theta \Theta} \geqslant \sigma_{\Theta D}$. Then, we will complete the proof proving that the convergence in $\sigma_{\Theta D}$ implies the one in $\sigma_{\Theta} \hat{\Theta}$.

Let $\left(f_{n}\right)_{n \in \mathbb{N}}$ be a sequence of functions in $E$ converging to some $f \in \mathfrak{L C}\left(\mathbb{R}^{2 N}, \mathbb{R}^{N}\right)$ with respect to $\sigma_{\Theta D}$. Fixed any interval $I=[p, q]$, with $p, q \in \mathbb{Q}$, we consider its partition in $m$ subintervals of the same length, that is, for $m \in \mathbb{N}$,

$$
I=\bigcup_{i=0}^{m-1} I_{i}, \quad \text { where } I_{i}=\left[p+i \frac{q-p}{m}, p+(i+1) \frac{q-p}{m}\right], i=0, \ldots, m-1 .
$$

Moreover, for any $y \in \widehat{\mathcal{K}}_{j}^{I}$, with $j \in \mathbb{N}$, and any $m \in \mathbb{N}$, let $\underline{y}^{m}: I \rightarrow \mathbb{R}^{N}$ and $\bar{y}^{m}: I \rightarrow \mathbb{R}^{N}$ be the simple functions defined by

$$
\underline{y}_{k}^{m}(t)=\sum_{i=0}^{m-1} \chi_{I_{i}}(t) \inf _{s \in I_{i}} y_{k}(s) \quad \text { and } \quad \bar{y}_{k}^{m}(t)=\sum_{i=0}^{m-1} \chi_{I_{i}}(t) \sup _{s \in I_{i}} y_{k}(s),
$$

where $\chi_{J}$ is the characteristic function of the interval $J$. Then, clearly one has

$$
\underline{y}^{m}(t) \leqslant y(t) \leqslant \bar{y}^{m}(t), \quad \text { for all } t \in I .
$$

Fix $0<\varepsilon<1 / 3$. For every $j \in \mathbb{N}$ there are $r_{j} \in \mathbb{N}$ and $z_{1}, \ldots, z_{r_{j}} \in D \cap B_{j+1}$ such that $B_{j+1} \subset \bigcup_{i=1}^{r_{j}} B_{\varepsilon}\left(z_{i}\right)$. On the other hand, by construction and since $\widehat{\mathcal{K}}_{j}^{I}$ is compact, there is an $m_{0} \in \mathbb{N}$ so that

$$
\left\|\underline{y}^{m_{0}}-y\right\|_{L^{\infty}(I)}<\varepsilon \quad \text { and } \quad\left\|\bar{y}^{m_{0}}-y\right\|_{L^{\infty}(I)}<\varepsilon, \quad \text { for all } y \in \hat{\mathcal{K}}_{j}^{I} .
$$

From this, for every $y \in \widehat{\mathcal{K}}_{j}^{I}$ there are simple functions $\underline{y}_{D}^{m_{0}}, \bar{y}_{D}^{m_{0}}: I \rightarrow\left\{z_{1}, \ldots, z_{r_{j}}\right\}$ such that, denoted by $v$ the unitary vector $v=(1 / \sqrt{N}, \ldots, 1 / \sqrt{N})$,

$$
\left|\left(\underline{y}^{m_{0}}(t)-\varepsilon v\right)-\underline{y}_{D}^{m_{0}}(t)\right|<\varepsilon \text { and }\left|\left(\bar{y}^{m_{0}}(t)+\varepsilon v\right)-\bar{y}_{D}^{m_{0}}(t)\right|<\varepsilon
$$

for each $t \in I$. One can easily check that

$$
\begin{aligned}
\underline{y}_{D}^{m_{0}}(t) & \leqslant \underline{y}^{m_{0}}(t) \leqslant y(t) \leqslant \bar{y}^{m_{0}}(t) \leqslant \bar{y}_{D}^{m_{0}}(t), \quad \text { for all } t \in I, \\
\left\|\underline{y}_{D}^{m_{0}}-y\right\|_{L^{\infty}(I)} & <3 \varepsilon \quad \text { and }\left\|\bar{y}_{D}^{m_{0}}-y\right\|_{L^{\infty}(I)}<3 \varepsilon, \quad \text { for all } y \in \widehat{\mathcal{K}}_{j}^{I} .
\end{aligned}
$$

Therefore, from Lemma 3.3 and using the convergence in $\sigma_{\Theta D}$, we have that there is an $n_{0}$ such that for each $n>n_{0}, i=0, \ldots, m_{0}-1$ and $k=1, \ldots, N$,

$$
\begin{aligned}
\sup _{x \in \mathcal{K}_{j}^{I}, y \in \hat{\mathcal{K}}_{j}^{I}} \int_{I_{i}}\left[\left(f_{n}\right)_{k}(t, x(t), y(t))-f_{k}(t, x(t), y(t))\right] d t & \\
\leqslant & \sup _{x \in \mathcal{K}_{j}^{I}, y \in \hat{\mathcal{K}}_{j}^{I}} \int_{I_{i}}\left[\left(f_{n}\right)_{k}\left(t, x(t), \bar{y}_{D}^{m_{0}}(t)\right)-f_{k}\left(t, x(t), \bar{y}_{D}^{m_{0}}(t)\right)\right] d t \\
& \quad+\sup _{x \in \mathcal{K}_{j}^{I}, y \in \hat{\mathcal{K}}_{j}^{I}} \int_{I_{i}}\left|f_{k}\left(t, x(t), \bar{y}_{D}^{m_{0}}(t)\right)-f_{k}(t, x(t), y(t))\right| d t \\
\leqslant & \frac{3 \varepsilon}{m_{0}}+\sup _{x \in \mathcal{K}_{j}^{I}, y \in \hat{\mathcal{K}}_{j}^{I}} \int_{I_{i}} l_{f}^{2(j+1)}(t)\left\|\bar{y}_{D}^{m_{0}}-y\right\|_{L^{\infty}(I)} d t \\
\leqslant & \frac{3 \varepsilon}{m_{0}}+3 \varepsilon \int_{I_{i}} l_{f}^{2(j+1)}(t) d t \leqslant \frac{3 \varepsilon}{m_{0}}+3 \varepsilon \int_{I} l_{f}^{2(j+1)}(t) d t .
\end{aligned}
$$


On the other hand, using $\underline{y}_{D}^{m_{0}}$ and with analogous reasoning, one also has that

$$
\sup _{x \in \mathcal{K}_{j}^{I}, y \in \hat{\mathcal{K}}_{j}^{I}} \int_{I_{i}}\left[f_{k}(t, x(t), y(t))-\left(f_{n}\right)_{k}(t, x(t), y(t))\right] d t \leqslant \frac{3 \varepsilon}{m_{0}}+3 \varepsilon \int_{I} l_{f}^{2(j+1)}(t) d t .
$$

Therefore, taking into account that $f_{n}$ and $f$ have $N$ components and there are $m_{0}$ subintervals $I_{i}$ whose union is $I$, the two previous chains of inequalities yield to

$$
\sup _{x \in \mathcal{K}_{j}^{I}, y \in \hat{\mathcal{K}}_{j}^{I}}\left|\int_{I}\left[f_{n}(t, x(t), y(t))-f(t, x(t), y(t))\right] d t\right| \leqslant 3 N \varepsilon+3 N \varepsilon \int_{I} l_{f}^{2(j+1)}(t) d t,
$$

and from the arbitrariness on $\varepsilon$ we obtain the convergence in $\sigma_{\Theta} \hat{\Theta}$, as claimed.

The proof for the strong topologies can be carried out similarly. Maintaining the previous notation and arguing again for each $k=1, \ldots, N$, we can write

$$
\begin{aligned}
\left|\left(f_{n}\right)_{k}(t, x(t), y(t))-f_{k}(t, x(t), y(t))\right| \leqslant & \left|\left(f_{n}\right)_{k}\left(t, x(t), \bar{y}_{D}^{m_{0}}(t)\right)-f_{k}(t, x(t), y(t))\right| \\
& +\left|\left(f_{n}\right)_{k}\left(t, x(t), \underline{y}_{D}^{m_{0}}(t)\right)-f_{k}(t, x(t), y(t))\right| .
\end{aligned}
$$

Then, we obtain the conclusion by using, for each one of the integrals of the terms on the right-hand side, the same chain of inequalities (3.6) employed before.

The importance of the equivalence of the topologies in obtaining the continuity of the skew-product semiflow generated by a set of Carathéodory differential equations has been extensively treated in [15]. In that work the equivalence was obtained via a thorough topological study of the $l$-bounds of a set of Carathéodory functions. Hence, it is not surprising that a new result of continuity can be achieved.

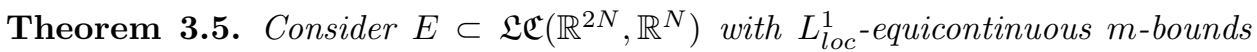
and such that every element of $E$ satisfies $\left(\mathbf{K}_{y}\right)$. Let $\Theta$ be the suitable set of moduli of continuity given by the $m$-bounds in Remark 2.13, $D$ a countable dense subset of $\mathbb{R}^{n}$, and $\sigma_{\Theta D}, \mathcal{T}_{\Theta D}$ the topologies of Definition 2.6.

(i) If $\left(\phi_{n}\right)_{n \in \mathbb{N}}$ is a sequence converging uniformly to $\phi$ in $\mathcal{C}$, and $\left(f_{n}\right)_{n \in \mathbb{N}}$ is a sequence in $E$ converging to $f$ in $\left(\mathfrak{L} \mathfrak{C}\left(\mathbb{R}^{2 N}, \mathbb{R}^{N}\right), \sigma_{\Theta D}\right)$, then, with the notation of Theorem 2.3, one has that

$$
x\left(\cdot, f_{n}, \phi_{n}\right) \stackrel{n \rightarrow \infty}{\longrightarrow} x(\cdot, f, \phi)
$$

uniformly in any $[-1, T] \subset I_{f, \phi}$.

(ii) if additionally $E$ is invariant with respect to the base flow, and $\bar{E}$ denotes the closure of $E$ in $\left(\mathfrak{L C}\left(\mathbb{R}^{2 N}, \mathbb{R}^{N}\right), \mathcal{T}\right)$, where $\mathcal{T} \in\left\{\sigma_{\Theta D}, \mathcal{T}_{\Theta D}\right\}$ then the map

$$
\Phi: \mathcal{U} \subset \mathbb{R}^{+} \times \bar{E} \times \mathcal{C} \rightarrow \bar{E} \times \mathcal{C}, \quad(t, f, \phi) \mapsto\left(f_{t}, x_{t}(\cdot, f, \phi)\right),
$$

is a continuous skew-product semiflow.

Proof. As regards (i), consider a sequence $\left(f_{n}\right)_{n \in \mathbb{N}}$ in $E$ converging to some $f \in$ $\bar{E}$ with respect to $\sigma_{\Theta D}$, a sequence $\left(\phi_{n}\right)_{n \in \mathbb{N}}$ converging to some $\phi$ in $\mathcal{C}$ endowed with the compact-open topology, and a sequence $\left(t_{n}\right)_{n \in \mathbb{N}}$ converging to some $t$ in $\mathbb{R}$. From Ascoli-Arzelá's theorem, there exists a common modulus of continuity $\theta_{0} \in C\left(\mathbb{R}^{+}, \mathbb{R}^{+}\right)$for $\left\{\phi_{n} \mid n \in \mathbb{N}\right\} \cup\{\phi\}$ and so one can construct the sets $\mathcal{F}_{0}$ and $\bar{\Theta}$ as in (2.5) and (2.6), respectively, and the topology $\sigma_{\Theta} \bar{\Theta}$ as in Definition 2.6. Thanks to Proposition 3.4, one has that $\left(f_{n}\right)_{n \in \mathbb{N}}$ converges to $f$ also with respect to $\sigma_{\Theta} \bar{\Theta}$. Therefore, one has the result using Theorem 2.15. 
On the other hand, (ii) is a consequence of (i), of Propositions 2.12 and 3.2, and of the continuity of the function $(t, f) \mapsto f_{t}$ proved in [15, Theorem 3.8].

As follows we relate the previous results to monotone skew-product semiflows. Recall the strongly ordering defined on $\mathcal{C}$ in (3.1).

Definition 3.6 (Monotone skew-product semiflow). Given a set $E \subset \mathfrak{L} \mathfrak{C}\left(\mathbb{R}^{2 N}, \mathbb{R}^{N}\right)$ invariant with respect to the base flow, the skew-product semiflow

$$
\Phi: \mathcal{U} \subset \mathbb{R}^{+} \times E \times \mathcal{C} \rightarrow E \times \mathcal{C}, \quad(t, f, \phi) \mapsto\left(f_{t}, x_{t}(\cdot, f, \phi)\right)
$$

is said to be monotone if

$$
x(t, f, \phi) \leqslant x(t, f, \psi) \quad \text { whenever } t \in I_{f, \phi} \cap I_{f, \psi}, f \in E \text { and } \phi \leqslant \psi .
$$

Monotone skew-product semiflows have been extensively studied in the literature. As follows, we show that the skew-product semiflow generated by a set of Carathéodory delay differential equations is monotone if and only if all the vector fields are cooperative.

Theorem 3.7. Consider $E \subset \mathfrak{L} \mathfrak{C}\left(\mathbb{R}^{2 N}, \mathbb{R}^{N}\right)$ invariant with respect to the base flow. The following statements are equivalent:

(a) Every $f \in E$ satisfies $\left(\mathbf{K}_{x}\right)$ and $\left(\mathbf{K}_{y}\right)$.

(b) The skew-product semiflow (3.7) is monotone.

Proof. $(\mathrm{a}) \Rightarrow(\mathrm{b})$ Consider $f \in E, \phi, \psi \in \mathcal{C}$ with $\phi \leqslant \psi$. Notice that $x(t, f, \phi)$ and $x(t, f, \psi)$ are the solutions of the Carathéodory ordinary differential problems

$$
\left\{\begin{array} { l } 
{ x ^ { \prime } ( t ) = f ( t , x , \phi ( t - 1 ) ) , } \\
{ x ( 0 ) = \phi ( 0 ) , }
\end{array} \quad \text { and } \quad \left\{\begin{array}{l}
x^{\prime}(t)=f(t, x, \psi(t-1)), \\
x(0)=\psi(0) .
\end{array}\right.\right.
$$

for $t \in[0,1]$, assuming that $[0,1] \subset I_{f, \phi} \cap I_{f, \psi}$. Then $x(t, f, \phi) \leqslant x(t, f, \psi)$ for each $t \in[0,1]$ follows from Walter [28, Theorem 2]. This argument can be iterated on successive intervals of length smaller than one to obtain the inequality in any compact interval contained in $I_{f, \phi} \cap I_{f, \psi}$.

$(\mathrm{b}) \Rightarrow(\mathrm{a})$ We prove $\left(\mathbf{K}_{x}\right)$. Consider $f \in E$, and $a, b, c \in \mathbb{R}^{N}$ such that $a \leqslant b$ and $a_{k}=b_{k}$ for some $k \in\{1, \ldots, N\}$. Fixed any $t_{0} \in \mathbb{R}$, consider $\phi, \psi \in \mathcal{C}\left(\left[t_{0}-1, t_{0}\right], \mathbb{R}^{N}\right)$ defined by $\phi(s)=\left(t_{0}-s\right) c+\left(s-t_{0}+1\right) a$ and $\psi(s)=\left(t_{0}-s\right) c+\left(s-t_{0}+1\right) b$, and let $x(t)$ and $y(t)$ be the solutions of the Carathéodory delay differential problems

$$
\left\{\begin{array} { l } 
{ x ^ { \prime } ( t ) = f ( t , x ( t ) , x ( t - 1 ) ) , } \\
{ x ( s ) = \phi ( s ) , \quad s \in [ t _ { 0 } - 1 , t _ { 0 } ] }
\end{array} \quad \text { and } \quad \left\{\begin{array}{l}
y^{\prime}(t)=f(t, y(t), y(t-1)), \\
y(s)=\psi(s), \quad s \in\left[t_{0}-1, t_{0}\right]
\end{array}\right.\right.
$$

defined on $\left[t_{0}, t_{0}+h\right]$ for some $h \in[0,1]$.

From $\phi(s) \leqslant \psi(s)$ for each $s \in\left[t_{0}-1, t_{0}\right]$ and (b) we deduce that $x(t) \leqslant y(t)$ for $t \in\left[t_{0}, t_{0}+h\right]$ which together with $x_{k}\left(t_{0}\right)=a_{k}=b_{k}=y_{k}\left(t_{0}\right)$ yields to

$$
\frac{1}{h} \int_{t_{0}}^{t_{0}+h} f_{k}(s, x(s), \phi(s-1)) d s \leqslant \frac{1}{h} \int_{t_{0}}^{t_{0}+h} f_{k}(s, y(s), \psi(s-1)) d s .
$$

Moreover, denoting by $M(h)=\max _{s \in\left[t_{0}, t_{0}+h\right]}(|x(s)-a|+|\phi(s-1)-c|)$ and by $l$ and adequate $l$-bound for $f$, one can easily prove that

$$
\left.\mid \frac{1}{h} \int_{t_{0}}^{t_{0}+h}\left(f_{k}(s, x(s), \phi(s-1))-f_{k}(s, a, c)\right)\right) d s \mid \leqslant M(h) \frac{1}{h} \int_{t_{0}}^{t_{0}+h} l(s) d s .
$$


However, thanks to Lebesgue's theorem, for a.e. $t_{0} \in \mathbb{R}$ the right-hand side of the previous inequality vanishes as $h \rightarrow 0$ since $\lim _{h \rightarrow 0} M(h)=0$. Therefore,

$$
\lim _{h \rightarrow 0} \frac{1}{h} \int_{t_{0}}^{t_{0}+h} f_{k}(s, x(s), \phi(s-1)) d s=f_{k}\left(t_{0}, a, c\right) \quad \text { for a.e. } t_{0} \in \mathbb{R} .
$$

Analogously, we have that for a.e. $t_{0} \in \mathbb{R}$ the integral on the right-hand side of (3.8) converges to $f_{k}\left(t_{0}, b, c\right)$ as $h \rightarrow 0$. Hence, as $h \rightarrow 0$, (3.8) becomes

$$
f_{k}\left(t_{0}, a, c\right) \leqslant f_{k}\left(t_{0}, b, c\right) \quad \text { for a.e. } t_{0} \in \mathbb{R}
$$

which concludes the proof of $\left(\mathbf{K}_{x}\right)$, as claimed.

In order to prove $\left(\mathbf{K}_{y}\right)$, take $f \in E$ and $a, b, c \in \mathbb{R}^{N}$ such that $b \leqslant c$. Fix $t_{0} \in \mathbb{R}$ and consider $\phi, \psi \in \mathcal{C}\left(\left[t_{0}-1, t_{0}\right], \mathbb{R}^{N}\right)$ defined by $\phi(s)=\left(t_{0}-s\right) b+\left(s-t_{0}+1\right) a$ and $\psi(s)=\left(t_{0}-s\right) c+\left(s-t_{0}+1\right) a$. Maintaining the above notation for $x(t)$ and $y(t)$ and taking into account that now $x\left(t_{0}\right)=y\left(t_{0}\right)=a$, we deduce (3.8) for all $k \in\{1, \ldots, N\}$, and Lebesgue's theorem provides

$$
f_{k}\left(t_{0}, a, b\right) \leqslant f_{k}\left(t_{0}, a, c\right), \quad \text { for all } k=1, \ldots, N \text {, and a.e. } t_{0} \in \mathbb{R},
$$

which finishes the proof.

As a consequence of this characterization and of Theorem 3.5, one immediately obtains that the monotone skew-product semiflow generated by a suitable set of Carathéodory delay differential equations is continuous.

Corollary 3.8. Consider $E \subset \mathfrak{L} \mathfrak{C}\left(\mathbb{R}^{2 N}, \mathbb{R}^{N}\right)$ invariant with respect to the base flow and with $L_{\text {loc }}^{1}$-equicontinuous $m$-bounds. Let $\bar{E}$ be its closure in $\mathfrak{L} \mathfrak{C}\left(\mathbb{R}^{2 N}, \mathbb{R}^{N}\right)$ with respect to $\mathcal{T} \in\left\{\sigma_{\Theta D}, \mathcal{T}_{\Theta D}\right\}$, where $\Theta$ is the suitable set of moduli of continuity given by the $m$-bounds in Remark 2.13 , and $D$ a countable dense subset of $\mathbb{R}^{N}$. If

$$
\Phi: \mathcal{U} \subset \mathbb{R}^{+} \times \bar{E} \times \mathcal{C} \rightarrow \bar{E} \times \mathcal{C}, \quad(t, f, \phi) \mapsto\left(f_{t}, x_{t}(\cdot, f, \phi)\right),
$$

is a monotone skew-product semiflow, then it is also continuous.

Proof. The result is a direct consequence of Theorem 3.5 and Theorem 3.7.

Next we want to remark that cooperative systems of Carathéodory differential equations preserve the strict order for each component.

Lemma 3.9. Assume that system (2.3) is cooperative, that is, $f$ satisfies Kamke's conditions $\left(\mathbf{K}_{x}\right)$ and $\left(\mathbf{K}_{y}\right)$. Consider two initial data $\phi$ and $\psi \in \mathcal{C}$ such that $\phi \leqslant \psi$ and $\phi_{k}(0)<\psi_{k}(0)$ for some $k \in\{1, \ldots, N\}$. Then

$$
x_{k}(t, f, \phi)<x_{k}(t, f, \psi) \quad \text { whenever } t>0 \text { and } t \in I_{f, \phi} \cap I_{f, \psi} .
$$

In particular, if $\phi(0) \ll \psi(0)$, then $x(t, f, \phi) \ll x(t, f, \psi)$.

Proof. We denote $v(t)=x(t, f, \phi)$ and $w(t)=x(t, f, \psi)$ and reason for the first component for simplicity of notation. The proof for the other components is analogous. We claim that $w_{1}^{\prime}(t)-v_{1}^{\prime}(t) \geqslant-l(t)\left(w_{1}(t)-v_{1}(t)\right)$ for an appropriate $l$-bound of $f$. From Theorem 3.7 we know that $w(t)-v(t) \geqslant 0$ for each $t \in I_{f, \phi} \cap I_{f, \psi}$. Then, from $\left(\mathbf{K}_{x}\right)$ we deduce that

$$
f_{1}(t, w(t), w(t-1)) \geqslant f_{1}\left(t, w_{1}(t), v_{2}(t), \ldots, v_{N}(t), v(t-1)\right),
$$

which together with the following inequality due to $(\mathbf{L})$

$$
f_{1}\left(t, w_{1}(t), v_{2}(t), \ldots, v_{N}(t), v(t-1)\right)-f_{1}(t, v(t), v(t-1) \geqslant-l(t)(w(t)-v(t))
$$


proves the claim. Therefore, recalling that $w_{1}(0)-v_{1}(0)=\psi_{1}(0)-\phi_{1}(0)>0$, and using a comparison result for Carathéodory differential equations [28, Theorem 2], we conclude that $w_{1}^{\prime}(t)-v_{1}^{\prime}(t) \geqslant\left(w_{1}(0)-v_{1}(0)\right) \exp \left(-\int_{0}^{t} l(s) d s\right)>0$ for each $t>0$ satisfying $t \in I_{f, \phi} \cap I_{f, \psi}$, which finishes the proof.

We conclude this section by considering two more sets of assumptions which still involve Kamke's conditions. Firstly, we will consider $\left(\mathbf{K}_{x}\right)$ and $\left(\mathbf{K}_{y}\right)$ together with a very mild assumption on the Lipschitz coefficients of the components of the vector field, and then a condition of monotonicity which implies both $\left(\mathbf{K}_{x}\right)$ and $\left(\mathbf{K}_{y}\right)$. It is worth noticing that, at least for the strong topologies, we are now able to obtain the same results as before but without assuming that the vector fields have $L_{l o c^{-}}^{1}$ equicontinuous $m$-bounds. In fact, we obtain something more. In both cases of strong and weak topologies we are able to prove the continuous dependence of the solutions and the continuity of the associated skew-product semiflow when weaker topologies are employed. To the aim let us recall such two further families of strong and weak topologies on $\mathfrak{S C}\left(\mathbb{R}^{2 N}, \mathbb{R}^{N}\right)$.

Definition 3.10 (Topologies $\mathcal{T}_{P}$ and $\sigma_{P}$ ). Let $P$ be a countable dense subset of $\mathbb{R}^{2 N}$. We call $\mathcal{T}_{P}\left(\right.$ resp. $\left.\sigma_{P}\right)$ the topology on $\mathfrak{S} \mathfrak{C}\left(\mathbb{R}^{2 N}, \mathbb{R}^{N}\right)$ generated by the family of seminorms

$$
p_{I, x}(f)=\int_{I}|f(t, x)| d t \quad\left(\text { resp. } p_{I, x}(f)=\left|\int_{I} f(t, x) d t\right|\right)
$$

for $f \in \mathfrak{S C}\left(\mathbb{R}^{2 N}, \mathbb{R}^{N}\right), x \in P, I=\left[q_{1}, q_{2}\right], q_{1}, q_{2} \in \mathbb{Q} . \quad\left(\mathfrak{S C}\left(\mathbb{R}^{2 N}, \mathbb{R}^{N}\right), \mathcal{T}_{P}\right)$ and $\left(\mathfrak{S C}\left(\mathbb{R}^{2 N}, \mathbb{R}^{N}\right), \sigma_{P}\right)$ are locally convex metric spaces.

Remark 3.11. Consider any two countable dense subsets $D$ and $D_{1}$ of $\mathbb{R}^{N}$ and the countable dense subset $P=D_{1} \times D$ of $\mathbb{R}^{2 N}$. Let $\Theta$ and $\widehat{\Theta}$ be any pair of suitable sets of moduli of continuity as in Definition 2.5, such that for any $I=\left[q_{1}, q_{2}\right]$, $q_{1}, q_{2} \in \mathbb{Q}$ and $j \in \mathbb{N}$ one has

$$
\theta_{j}^{I}(t) \leqslant \hat{\theta}_{j}^{I}(t), \quad \text { for all } t \in[0, \infty) .
$$

Then, one can draw the following chains of order:

$\sigma_{P} \leqslant \mathcal{T}_{P} \leqslant \mathcal{T}_{\Theta D} \leqslant \mathcal{T}_{\Theta} \leqslant \mathcal{T}_{\Theta \Theta} \leqslant \mathcal{T}_{\Theta \Theta}$ and $\sigma_{P} \leqslant \sigma_{\Theta D} \leqslant \sigma_{\Theta} \leqslant \sigma_{\Theta \Theta} \leqslant \sigma_{\Theta} \hat{\Theta} \leqslant \mathcal{T}_{\Theta \Theta}$,

where, in particular, the order relations $\mathcal{T}_{\Theta} \leqslant \mathcal{T}_{\Theta \Theta}$ and $\sigma_{\Theta} \leqslant \sigma_{\Theta \Theta}$ hold true thanks to Proposition 2.13 in [15]. Clearly, one might expand the previous chains of inequalities (or generate new branches) by considering more suitable sets of moduli of continuity (satisfying appropriate relations of partial order) and/or different countable dense subsets of $\mathbb{R}^{N}$ and $\mathbb{R}^{2 N}$, and the corresponding induced topologies.

Recall that a set $S$ of positive functions is $L_{l o c}^{1}$-bounded if $\sup _{u \in S} \int_{-r}^{r} u(t) d t<\infty$ for each $r>0$.

Definition 3.12. A set $E \subset \mathfrak{L} \mathfrak{C}\left(\mathbb{R}^{2 N}, \mathbb{R}^{N}\right)$ satisfies

$\left(\mathbf{L}_{x}\right)$ if for each $f \in E, j \in \mathbb{N}$ and $k=1, \ldots, N$ there is $u_{f_{k}}^{j} \in L_{l o c}^{1}$ such that, for any $(x, y)=\left(x_{1}, \ldots, x_{k}, \ldots, x_{N}, y\right),\left(\bar{x}^{k}, y\right)=\left(x_{1} \ldots, \bar{x}_{k}, \ldots, x_{N}, y\right) \in B_{j} \subset \mathbb{R}^{2 N}$, $\left|f_{k}(t, x, y)-f_{k}\left(t, \bar{x}^{k}, y\right)\right| \leqslant u_{f_{k}}^{j}(t)\left|x_{k}-\bar{x}_{k}\right|, \quad$ for a.e. $t \in \mathbb{R}$, and the set $\left\{u_{f_{k}}^{j} \mid f \in E\right\}$ is $L_{l o c}^{1}$-bounded. 
Notice that this assumption is formulated componentwise and then is weaker that the existence of $L_{l o c}^{1}$-bounded $l$-bounds and $L_{l o c}^{1}$-bounded $l_{1}$-bounds (see Definitions 2.1 and 2.4).

The following result gathers together two auxiliary facts concerning $\left(\mathbf{L}_{x}\right)$.

Proposition 3.13. Let $P$ be a countable dense subset of $\mathbb{R}^{2 N}, E$ be a subset of $\mathfrak{L C}\left(\mathbb{R}^{2 N}, \mathbb{R}^{N}\right)$ satisfying $\left(\mathbf{L}_{x}\right)$, and $\mathcal{T} \in\left\{\mathcal{T}_{P}, \sigma_{P}\right\}$ as in Definition 3.10. Then

(i) the closure of $E$ with respect to $\mathcal{T}$ satisfies $\left(\mathbf{L}_{x}\right)$;

(ii) if every function in $E$ satisfies also $\left(\mathbf{K}_{x}\right),\left(\mathbf{K}_{y}\right)$, and $\Theta, \widehat{\Theta}$ is any pair of suitable sets of moduli of continuity as in Definition 2.5, then

$$
\operatorname{cls}_{\left(\mathfrak{L C}, \sigma_{\Theta} \widehat{\Theta}\right)}(E)=\operatorname{cls}_{\left(\mathfrak{L C}, \sigma_{P}\right)}(E) \quad \text { and } \quad \operatorname{cls}_{\left(\mathfrak{L C}, \mathcal{T}_{\Theta \widehat{\Theta}}\right)}(E)=\operatorname{cls}_{\left(\mathfrak{L C}, \mathcal{T}_{P}\right)}(E) .
$$

Proof. The proof of (i) can be carried out following exactly the same arguments of [15, Proposition 2.19].

As regards (ii), Proposition 3.4 serves as a guide for the proof and we will only remark the differences between them. Considered $P \subset \mathbb{R}^{2 N}$ as in the assumptions, and keeping in mind Remark 3.11, we shall prove that the convergence in $\sigma_{P}$ implies the one in $\sigma_{\Theta} \hat{\Theta}$ for any pair of suitable sets of moduli of continuity $\Theta, \widehat{\Theta}$.

Let $\left(f_{n}\right)_{n \in \mathbb{N}}$ be a sequence of functions in $E$ converging to some $f \in \mathfrak{L} \mathfrak{C}\left(\mathbb{R}^{2 N}, \mathbb{R}^{N}\right)$ with respect to $\sigma_{P}$ and fix any interval $I=[p, q]$, with $p, q \in \mathbb{Q}$ and any $j \in \mathbb{N}$. As in Proposition 3.4, given $\varepsilon>0, x \in \mathcal{K}_{j}^{I}$ and $y \in \widehat{\mathcal{K}}_{j}^{I}$, there is an $m_{0} \in \mathbb{N}$ for which we construct simple functions $\underline{x}_{P}^{m_{0}}, \bar{x}_{P}^{m_{0}}, \underline{y}_{P}^{m_{0}}, \bar{y}_{P}^{m_{0}}: I \rightarrow P$ such that

$$
\underline{x}_{P}^{m_{0}}(t) \leqslant x(t) \leqslant \bar{x}_{P}^{m_{0}}(t) \quad \text { and } \quad \underline{y}_{P}^{m_{0}}(t) \leqslant y(t) \leqslant \bar{y}_{P}^{m_{0}}(t) \quad \text { for all } t \in I,
$$

approaching $x$ and $y$ in $L^{\infty}(I)$, uniformly on $\mathcal{K}_{j}^{I}$ and $\widehat{\mathcal{K}}_{j}^{I}$ respectively.

Next we can proceed as in the proof of Proposition 3.4. First we will denote

$$
\begin{aligned}
\bar{x}_{P}^{m_{0}}(t) & =\left(\left(\bar{x}_{P}^{m_{0}}\right)_{1}(t), \ldots,\left(\bar{x}_{P}^{m_{0}}\right)_{k}(t), \ldots,\left(x_{P}^{m_{0}}\right)_{N}(t)\right), \\
\left(\widehat{x}_{P}^{m_{0}}\right)^{k}(t) & =\left(\left(\bar{x}_{P}^{m_{0}}\right)_{1}(t), \ldots, x_{k}(t), \ldots,\left(x_{P}^{m_{0}}\right)_{N}(t)\right) .
\end{aligned}
$$

From Lemma 3.3 we deduce that $\left(f_{n}\right)_{k}(t, x(t), y(t)) \leqslant\left(f_{n}\right)_{k}\left(t,\left(\widehat{x}_{P}^{m_{0}}\right)^{k}(t), \bar{y}_{P}^{m_{0}}(t)\right)$, an hence, now instead of (3.6), we will obtain

$$
\begin{aligned}
& \sup _{x \in \mathcal{K}_{j}^{I}, y \in \hat{\mathcal{K}}_{j}^{I}} \int_{I_{i}}\left[\left(f_{n}\right)_{k}(t, x(t), y(t))-f_{k}(t, x(t), y(t))\right] d t \\
& \leqslant \sup _{x \in \mathcal{K}_{j}^{I}, y \in \hat{\mathcal{K}}_{j}^{I}} \int_{I_{i}}\left|\left(f_{n}\right)_{k}\left(t,\left(\widehat{x}_{P}^{m_{0}}\right)^{k}(t), \bar{y}_{P}^{m_{0}}(t)\right)-\left(f_{n}\right)_{k}\left(t, \bar{x}_{P}^{m_{0}}(t), \bar{y}_{P}^{m_{0}}(t)\right)\right| d t \\
& \quad+\sup _{x \in \mathcal{K}_{j}^{I}, y \in \hat{\mathcal{K}}_{j}^{I}} \int_{I_{i}}\left[\left(f_{n}\right)_{k}\left(t, \bar{x}_{P}^{m_{0}}(t), \bar{y}_{P}^{m_{0}}(t)\right)-f_{k}\left(t, \bar{x}_{P}^{m_{0}}(t), \bar{y}_{P}^{m_{0}}(t)\right)\right] d t \\
& \quad+\sup _{x \in \mathcal{K}_{j}^{I}, y \in \hat{\mathcal{K}}_{j}^{I}} \int_{I_{i}}\left|f_{k}\left(t, \bar{x}_{P}^{m_{0}}(t), \bar{y}_{P}^{m_{0}}(t)\right)-f_{k}(t, x(t), y(t))\right| d t \\
& \leqslant \sup _{x \in \mathcal{K}_{j}^{I}} \int_{I_{i}} u_{\left(f_{n}\right)_{k}}^{2(j+1)}(t)\left\|\left(\bar{x}_{P}^{m_{0}}\right)_{k}-x_{k}\right\|_{L^{\infty}(I)} d t+\frac{3 \varepsilon}{m_{0}} \\
& \quad+\sup _{y \in \hat{\mathcal{K}}_{j}^{I}} \int_{I_{i}} l_{f}^{2(j+1)}(t)\left\|\bar{y}_{P}^{m_{0}}-y\right\|_{L^{\infty}(I)} d t \leqslant \frac{3 \varepsilon}{m_{0}}+3 \varepsilon \int_{I_{i}}\left[u_{\left(f_{n}\right)_{k}}^{2(j+1)}(t)+l_{f}^{2(j+1)}(t)\right] d t .
\end{aligned}
$$


The rest of the proof is exactly as in Proposition 3.4, once one realizes that, due to the assumption of $L_{l o c}^{1}$-boundedness for $\left\{u_{g}^{2(j+1)} \mid g \in E\right\}$, the integral on the right-hand side of the previous inequality is bounded uniformly on $n \in \mathbb{N}$. The proof for $\mathcal{T}_{P}$ and $\mathcal{T}_{\Theta} \hat{\Theta}$ is similar and again on the line of the suggestion given in Proposition 3.4.

We are now ready to give the first result of continuous dependence of the solutions employing the topologies $\mathcal{T}_{P}$ and $\sigma_{P}$, and of continuity of the induced skew-product semiflows.

Theorem 3.14. Let $E$ be a subset of $\mathfrak{L}\left(\mathbb{R}^{2 N}, \mathbb{R}^{N}\right)$ satisfying $\left(\mathbf{L}_{x}\right)$, and such that each element of $E$ satisfies $\left(\mathbf{K}_{x}\right)$ and $\left(\mathbf{K}_{y}\right)$. Let $P$ be a countable dense subset of $\mathbb{R}^{2 N}$ and consider the topologies $\sigma_{P}, \mathcal{T}_{P}$ as constructed in Definition 3.10.

(i) If $\left(\phi_{n}\right)_{n \in \mathbb{N}}$ is a sequence in $\mathcal{C}$ converging uniformly to $\phi \in \mathcal{C}$, and $\left(f_{n}\right)_{n \in \mathbb{N}}$ is a sequence in $E$ converging to $f$ in $\left(\mathfrak{L} \mathfrak{C}\left(\mathbb{R}^{2 N}, \mathbb{R}^{N}\right), \mathcal{T}_{P}\right)$, then, with the notation of Theorem 2.3, one has that

$$
x\left(\cdot, f_{n}, \phi_{n}\right) \stackrel{n \rightarrow \infty}{\longrightarrow} x(\cdot, f, \phi)
$$

uniformly in any $[-1, T] \subset I_{f, \phi}$.

(ii) if $E$ is invariant with respect to the base flow, and $\bar{E}$ denotes the closure of $E$ in $\left(\mathfrak{L} \mathfrak{C}\left(\mathbb{R}^{2 N}, \mathbb{R}^{N}\right), \mathcal{T}_{P}\right)$ then the map

$$
\Phi: \mathcal{U} \subset \mathbb{R}^{+} \times \bar{E} \times \mathcal{C} \rightarrow \bar{E} \times \mathcal{C}, \quad(t, f, \phi) \mapsto\left(f_{t}, x_{t}(\cdot, f, \phi)\right),
$$

is a local monotone continuous skew-product semiflow.

(iii) If additionally $E$ has $L_{l o c}^{1}$-equicontinuous $m$-bounds, then (i) and (ii) hold true also when the topology $\sigma_{P}$ is employed in place of $\mathcal{T}_{P}$.

Proof. In order to prove (i), first consider $t \in[0,1]$ and the Carathéodory ordinary differential equations Cauchy problems

$$
\left\{\begin{array} { l } 
{ x ^ { \prime } ( t ) = f _ { n } ( t , x ( t ) , \phi _ { n } ( t - 1 ) ) } \\
{ x ( 0 ) = \phi _ { n } ( 0 ) . }
\end{array} \text { and } \quad \left\{\begin{array}{l}
x^{\prime}(t)=f(t, x(t), \phi(t-1)) \\
x(0)=\phi(0) .
\end{array}\right.\right.
$$

We shall prove the uniform convergence of $\left(x\left(\cdot, f_{n}, \phi_{n}\right)\right)_{n \in \mathbb{N}}$ to $x(\cdot, f, \phi)$ in $[0, T]$ for any $0<T<\min \left\{1, b_{f, \phi}\right\}$. Denote

$$
0<\rho=1+\max \left\{\left(\left\|\phi_{n}\right\|_{\mathcal{C}}\right)_{n \in \mathbb{N}},\|x(\cdot, f, \phi)\|_{\mathcal{C}([-1, T])}\right\},
$$

and for all $n \in \mathbb{N}$ define

$$
z_{n}(t)= \begin{cases}x\left(t, f_{n}, \phi_{n}\right), & \text { if } 0 \leqslant t<T_{n}, \\ x\left(T_{n}, f_{n}, \phi_{n}\right), & \text { if } T_{n} \leqslant t \leqslant T .\end{cases}
$$

where $T_{n}=\sup \left\{t \in[0, T]|| x\left(s, f_{n}, \phi_{n}\right) \mid \leqslant \rho, \forall s \in[0, t]\right\}$. From (3.10) and the continuity of $\left(x\left(\cdot, f_{n}, \phi_{n}\right)\right)_{n \in \mathbb{N}}$, we deduce that $T_{n}>0$ for any $n \in \mathbb{N}$. In particular, $\left(z_{n}\right)_{n \in \mathbb{N}}$ is uniformly bounded. Consider $j \in \mathbb{N}$ so that $2 \rho<j$. Now we want to control $\left|z_{n}\left(t_{1}\right)-z_{n}\left(t_{2}\right)\right|$ for $t_{1}, t_{2} \in\left[0, T_{n}\right)$ and $t_{1}<t_{2}$. For simplicity of notation, we will just study the first of the components:

$$
\left|\left(z_{n}\right)_{1}\left(t_{1}\right)-\left(z_{n}\right)_{1}\left(t_{2}\right)\right| \leqslant \int_{t_{1}}^{t_{2}}\left|\left(f_{n}\right)_{1}\left(s, z_{n}(s), \phi_{n}(s-1)\right)\right| d s .
$$


It is easy to check that there is a $k_{j}>j$ and $a, b \in P \cap B_{k_{j}}$ such that $a \leqslant(x, y) \leqslant b$ for each $(x, y) \in B_{j}$. From $\left(\mathbf{K}_{x}\right)$ and $\left(\mathbf{K}_{y}\right)$ we obtain

$$
\begin{aligned}
\left(f_{n}\right)_{1}\left(s,\left(z_{n}\right)_{1}(s), a_{2}, \ldots, a_{2 N}\right) \leqslant\left(f_{n}\right)_{1}\left(s, z_{n}(s)\right. & \left., \phi_{n}(s-1)\right) \\
& \leqslant\left(f_{n}\right)_{1}\left(s,\left(z_{n}\right)_{1}(s), b_{2}, \ldots, b_{2 N}\right) .
\end{aligned}
$$

Thus, denoting by $g, g_{n}, h$ and $h_{n}: \mathbb{R} \rightarrow \mathbb{R}$ the functions of $\mathfrak{L} \mathfrak{C}(\mathbb{R}, \mathbb{R})$ given by

$$
\begin{aligned}
& g(t, v)=(f)_{1}\left(t, v, a_{2} \ldots, a_{2 N}\right) \quad \text { and } \quad g_{n}(t, v)=\left(f_{n}\right)_{1}\left(t, v, a_{2} \ldots, a_{2 N}\right) \\
& h(t, v)=(f)_{1}\left(t, v, b_{2} \ldots, b_{2 N}\right) \quad \text { and } \quad h_{n}(t, v)=\left(f_{n}\right)_{1}\left(t, v, b_{2} \ldots, b_{2 N}\right),
\end{aligned}
$$

we deduce that $\left|\left(f_{n}\right)_{1}\left(s, z_{n}(s), \phi_{n}(s-1)\right)\right| \leqslant\left|g_{n}\left(s,\left(z_{n}\right)_{1}(s)\right)\right|+\left|h_{n}\left(s,\left(z_{n}\right)_{1}(s)\right)\right|$ and

$$
\begin{aligned}
\left|\left(z_{n}\right)_{1}\left(t_{1}\right)-\left(z_{n}\right)_{1}\left(t_{2}\right)\right| \leqslant \int_{t_{1}}^{t_{2}}\left|g_{n}\left(s,\left(z_{n}\right)_{1}(s)\right)-g\left(s,\left(z_{n}\right)_{1}(s)\right)\right| d s & \\
& +\int_{t_{1}}^{t_{2}}\left|h_{n}\left(s,\left(z_{n}\right)_{1}(s)\right)-h\left(s,\left(z_{n}\right)_{1}(s)\right)\right| d s \\
& +2 \int_{t_{1}}^{t_{2}} m_{f}^{k_{j}}(s) d s=I_{1}+I_{2}+I_{3}
\end{aligned}
$$

where $m_{f}^{k_{j}}$ is the optimal $m$-bound for $f$ on $B_{k_{j}} \subset \mathbb{R}^{2 N}$.

Let $D_{1}$ and $D_{2}$ be countable dense subsets of $\mathbb{R}$ and $\mathbb{R}^{2 N-1}$ such that $a_{1}, b_{1} \in D_{1}$ and $\left(a_{2}, \ldots, a_{2 N}\right),\left(b_{2}, \ldots, b_{2 N}\right) \in D_{2}$. Since $\left(f_{n}\right)_{n \in \mathbb{N}}$ converges to $f$ with respect to $\mathcal{T}_{P}$ and the assumptions of Proposition 3.13 are satisfied, then $\left(f_{n}\right)_{n \in \mathbb{N}}$ converges to $f$ also with respect to $\mathcal{T}_{D_{1} \times D_{2}}$. From this fact one easily obtains that $\left(g_{n}\right)_{n \in \mathbb{N}}$ converges to $g$, and $h_{n}$ converges to $h$ with respect to $\mathcal{T}_{D_{1}}$.

Moreover, from $\left(\mathbf{L}_{x}\right)$ the sequence of functions $\left\{g_{n} \mid n \in \mathbb{N}\right\}$ and $\left\{h_{n} \mid n \in \mathbb{N}\right\}$ have $L_{l o c}^{1}$-bounded $l$-bounds, and consequently, thanks to Theorem 4.12 in [13] they also converge to $g$ and $h$ respectively with respect to the topology $\mathcal{T}_{B}$. In particular, this fact implies that the integrals $I_{1}$ and $I_{2}$ of (3.11) tend to 0 as $n$ goes to $\infty$ (see [13] for details about the convergence in $\mathcal{T}_{B}$ ). On the other hand, thanks to the absolute continuity of the Lebesgue integral, given $\varepsilon>0$ there is a $\delta>0$ such that $I_{3} \leqslant \varepsilon$ provided that $t_{2}-t_{1}<\delta$.

Summing up, and taking into account that in $\left[T_{n}, T\right]$ the left-hand side of (3.11) vanishes, given $\varepsilon>0$ there is a $\delta>0$ and an $n_{1} \in \mathbb{N}$ such that

$$
\left|\left(z_{n}\right)_{1}\left(t_{1}\right)-\left(z_{n}\right)_{1}\left(t_{2}\right)\right| \leqslant 3 \varepsilon \text { whenever } n \geqslant n_{1}, 0 \leqslant t_{1} \leqslant t_{2} \leqslant T \text { and } t_{2}-t_{1}<\delta,
$$

from which the equicontinuity of the sequence $\left(z_{n}\right)_{n \in \mathbb{N}}$ is obtained. Therefore, Ascoli-Arzelá's theorem implies that $\left(z_{n}\right)_{n \in \mathbb{N}}$ converges uniformly, up to a subsequence, to some continuous function $z:[0, T] \rightarrow \mathbb{R}^{N}$.

Next, we prove that $z(t) \equiv x(t, f, \phi)$ for each $t \in[0, T]$. Define

$$
T_{0}=\sup \{t \in[0, T]|| z(s) \mid<\rho-1 / 2 \quad \text { for all } s \in[0, t]\},
$$

and notice that $T_{0}>0$ because $\left(\phi_{n}\right)_{n \in \mathbb{N}}$ converges uniformly to $\phi$ in $[-1,0]$ and $z$ is continuous. Since $z_{n}$ converges uniformly to $z$ in $[0, T]$, then there exists an $n_{0} \in \mathbb{N}$ such that if $n>n_{0}$, then $\left|z_{n}(t)\right|<\rho-1 / 4$ for all $t \in\left[0, T_{0}\right]$.

Therefore, for any $t \in\left[0, T_{0}\right]$ and for any $n>n_{0}$ one has $z_{n}(t)=x\left(t, f_{n}, \phi_{n}\right)$ and

$$
z_{n}(t)=\phi_{n}(0)+\int_{0}^{t} f_{n}\left(s, z_{n}(s), \phi_{n}(s-1)\right) d s, \quad t \in\left[0, T_{0}\right], n>n_{0} .
$$


Now consider the compact set $\mathcal{K}=\left\{z_{n} \mid n \in \mathbb{N}\right\} \cup\{z\} \subset \mathcal{C}\left([0, T], \mathbb{R}^{N}\right)$ and let $\theta: \mathbb{R}^{+} \rightarrow \mathbb{R}^{+}$be its modulus of continuity. Moreover, since $\left(\phi_{n}\right)_{n \in \mathbb{N}}$ converges uniformly to $\phi \in \mathcal{C}$, the set $\left\{\phi_{n} \mid n \in \mathbb{N}\right\} \cup\{\phi\}$ also has a common modulus of continuity, say $\hat{\theta}: \mathbb{R}^{+} \rightarrow \mathbb{R}^{+}$. The sets $\Theta=\{\theta\}$ and $\hat{\Theta}=\{\hat{\theta}\}$ are suitable sets of moduli of continuity. Then again thanks to Proposition 3.13, since $\left(f_{n}\right)_{n \in \mathbb{N}}$ converges to $f$ in $\left(\mathfrak{L C}\left(\mathbb{R}^{2 N}, \mathbb{R}^{N}\right), \mathcal{T}_{P}\right)$, then $\left(f_{n}\right)_{n \in \mathbb{N}}$ converges to $f$ also with respect to $\mathcal{T}_{\Theta} \hat{\Theta}$, and passing to the limit as $n \rightarrow \infty$ in (3.13), we deduce that

$$
z(t)=x_{0}+\int_{0}^{t} f(s, z(s), z(s-1)) d s, \quad t \in\left[0, T_{0}\right] .
$$

In other words, in $\left[0, T_{0}\right], z(t)$ is the solution of the problem on the right-hand side of (3.9). Finally, notice that it must be $T_{0}=T$. Otherwise, by (3.12) and by the continuity of $z$, one would have $\left|z\left(T_{0}\right)\right|=\left|x\left(T_{0}, f, x_{0}\right)\right|=\rho-1 / 2$, which contradicts (3.10). Hence, for any $t \in[0, T]$ we have that $x\left(t, f, x_{0}\right)=z(t)$ and $x\left(t, f_{n}, x_{0, n}\right)=z_{n}(t)$ for any $n \in \mathbb{N}$. This reasoning can be iterated to obtain the uniform convergence on any compact interval of the maximal interval of definition of $x\left(\cdot, f, x_{0}\right)$, which concludes the proof of (i).

We obtain (ii) as a consequence of (i), of Propositions 3.2 and 3.13, and of the continuity of the function $(t, f) \mapsto f_{t}$ proved in [15, Theorem 3.8].

As regards (iii), one can proceed exactly as for (i) except that now the equicontinuity of the sequence $\left(z_{n}\right)_{n \in \mathbb{N}}$ follows from the $L_{l o c}^{1}$-equicontinuity of the $m$-bounds. Notice that in this case, the limit as $n \rightarrow \infty$ in (3.13) is initially taken only for $t \in \mathbb{Q}$ but then one gets the result for any $t \in\left[0, T_{0}\right]$ thanks to the continuity of $z$ and of the integral operator. The result for the skew-product semiflow is obtained once again combining the continuous dependence of the solutions with the continuity of the function $(t, f) \mapsto f_{t}$ proved in [15, Theorem 3.8].

We introduce a stronger monotonicity condition implying both $\left(\mathbf{K}_{x}\right)$ and $\left(\mathbf{K}_{y}\right)$.

Definition 3.15. A function $f \in \mathfrak{L} \mathfrak{C}\left(\mathbb{R}^{2 N}, \mathbb{R}^{N}\right)$ satisfies

$\left(\mathbf{K}_{x y}\right)$ if for any $a, b, c, d \in \mathbb{R}^{N}$ with $a \leqslant b$ and $c \leqslant d$, then

$$
f_{k}(t, a, c) \leqslant f_{k}(t, b, d), \quad \text { for all } k=1, \ldots, N \text {, and a.e. } t \in \mathbb{R} .
$$

The following result gathers together some of the analogous properties proved for $\left(\mathbf{K}_{y}\right)$ before.

Proposition 3.16. Let $P$ be a countable dense set of $\mathbb{R}^{2 N}$ and $\mathcal{T} \in\left\{\mathcal{T}_{P}, \sigma_{P}\right\}$.

(i) If $f \in \mathfrak{L C}\left(\mathbb{R}^{2 N}, \mathbb{R}^{N}\right)$ satisfies $\left(\mathbf{K}_{x y}\right)$, then for any interval $I \subset \mathbb{R}$ and any $x, y, u, v \in \mathcal{C}\left(I, \mathbb{R}^{N}\right)$, with $x(t) \leqslant u(t)$ and $y(t) \leqslant v(t)$ for all $t \in I$, one has that

$$
f(t, x(t), y(t)) \leqslant f(t, u(t), v(t)), \quad \text { for a.e. } t \in I .
$$

(ii) If every function in $E \subset \mathfrak{L} \mathfrak{C}\left(\mathbb{R}^{2 N}, \mathbb{R}^{N}\right)$ satisfies $\left(\mathbf{K}_{x y}\right)$, then also any function in the closure of $E$ with respect to $\mathcal{T}$ satisfies $\left(\mathbf{K}_{x y}\right)$.

(iii) If every function in $E \subset \mathfrak{L} \mathfrak{C}\left(\mathbb{R}^{2 N}, \mathbb{R}^{N}\right)$ satisfies $\left(\mathbf{K}_{x y}\right)$, and $\Theta, \widehat{\Theta}$ is any pair of suitable sets of moduli of continuity as in Definition 2.5, then

$$
\operatorname{cls}_{\left(\mathfrak{L C}, \sigma_{\Theta} \widehat{\Theta}\right)}(E)=\operatorname{cls}_{\left(\mathfrak{L C}, \sigma_{P}\right)}(E) \quad \text { and } \quad \operatorname{cls}_{\left(\mathfrak{L C}, \mathcal{T}_{\Theta \widehat{\Theta}}\right)}(E)=\operatorname{cls}_{\left(\mathfrak{L C}, \mathcal{T}_{P}\right)}(E) .
$$

Proof. The statements can be proved using arguments similar to the ones employed in the proofs of Lemma 3.3, Proposition 3.2 and Proposition 3.4, respectively. 
Then the following additional result of continuity of the skew-product semiflows generated by a set of Carathéodory delay differential equations are obtained.

Theorem 3.17. Consider $E \subset \mathfrak{L} \mathfrak{C}\left(\mathbb{R}^{2 N}, \mathbb{R}^{N}\right)$ such that every element of $E$ satisfies $\left(\mathbf{K}_{x y}\right)$. Let $P$ be any countable dense subset of $\mathbb{R}^{2 N}$ and $\sigma_{P}, \mathcal{T}_{P}$ the topologies introduced in Definition 3.10.

(i) If $\left(\phi_{n}\right)_{n \in \mathbb{N}}$ is a sequence converging uniformly to $\phi$ in $\mathcal{C}$, and $\left(f_{n}\right)_{n \in \mathbb{N}}$ is a sequence in $E$ converging to $f$ in $\left(\mathfrak{L} \mathfrak{C}\left(\mathbb{R}^{2 N}, \mathbb{R}^{N}\right), \mathcal{T}_{P}\right)$, then, with the notation of Theorem 2.3, one has that

$$
x\left(\cdot, f_{n}, \phi_{n}\right) \stackrel{n \rightarrow \infty}{\longrightarrow} x(\cdot, f, \phi)
$$

uniformly in any $[-1, T] \subset I_{f, \phi}$.

(ii) if $E$ is invariant with respect to the base flow, and $\bar{E}$ denotes the closure of $E$ in $\left(\mathfrak{L} \mathfrak{C}\left(\mathbb{R}^{2 N}, \mathbb{R}^{N}\right), \mathcal{T}_{P}\right)$ then the map

$$
\Phi: \mathcal{U} \subset \mathbb{R}^{+} \times \bar{E} \times \mathcal{C} \rightarrow \bar{E} \times \mathcal{C}, \quad(t, f, \phi) \mapsto\left(f_{t}, x_{t}(\cdot, f, \phi)\right),
$$

is a local monotone continuous skew-product semiflow

(iii) If additionally $E$ has $L_{l o c}^{1}$-equicontinuous m-bounds, then (i) and (ii) hold true also when the topology $\sigma_{P}$ is employed in place of $\mathcal{T}_{P}$.

Proof. The proof can be carried out reasoning as in Theorem 3.14. Maintaining the notation of its proof, from $\left(\mathbf{K}_{x y}\right)$ we deduce the inequality

$$
\begin{aligned}
\left|\left(z_{n}\right)_{k}\left(t_{1}\right)-\left(z_{n}\right)_{k}\left(t_{2}\right)\right| \leqslant & \int_{t_{1}}^{t_{2}}\left|\left(f_{n}\right)_{k}\left(s, z_{n}(s), \phi_{n}(s-1)\right)\right| d s \\
\leqslant & \int_{t_{1}}^{t_{2}}\left|\left(f_{n}\right)_{k}(s, a)-(f)_{k}(s, a)\right| d s \\
& \quad+\int_{t_{1}}^{t_{2}}\left|\left(f_{n}\right)_{k}(s, b)-(f)_{k}(s, b)\right| d s+2 \int_{t_{1}}^{t_{2}} m_{f}^{j+1}(s) d s
\end{aligned}
$$

for $k=1, \ldots, N$, which, from the convergence of $\left(f_{n}\right)_{n \in \mathbb{N}}$ to $f$ with respect to $\mathcal{T}_{P}$ and the absolute continuity of the Lebesgue integral, leads to the equicontinuity of $\left(z_{n}\right)_{n \in \mathbb{N}}$. The rest of the proof follows exactly the same arguments with the help, in this case, of Proposition 3.16.

The reasoning behind the proofs of Theorems 3.17 and 3.14 immediately suggests that if one deals with a set of ordinary differential equation of Carathéodory type, two new results of continuity for the associated skew-product flow can be derived. Given $f \in \mathfrak{L} \mathfrak{C}\left(\mathbb{R}^{N}, \mathbb{R}^{N}\right)$ and $x_{0} \in \mathbb{R}^{N}$, we will denote by $x\left(\cdot, f, x_{0}\right): I_{f, x_{0}} \rightarrow \mathbb{R}^{N}$ the unique maximal solution of the Cauchy problem

$$
x^{\prime}(t)=f(t, x(t)), x(0)=x_{0} .
$$

The monotoniticy conditions, as well as the corresponding condition $\left(\mathbf{L}_{x}\right)$ for a set of functions in this case, are stated in the next definitions.

Definition 3.18. We say that $f \in \mathfrak{L C}\left(\mathbb{R}^{N}, \mathbb{R}^{N}\right)$ satisfies

$\left(\mathbf{K}_{1}\right)$ if for any $a, b \in \mathbb{R}^{N}$ with $a \leqslant b$ and $a_{k}=b_{k}$ for some $k \in\{1, \ldots, N\}$, then

$$
f_{k}(t, a) \leqslant f_{k}(t, b), \quad \text { for a.e. } t \in \mathbb{R}
$$


$\left(\mathbf{K}_{2}\right)$ if for any $a, b \in \mathbb{R}^{N}$ with $a \leqslant b$, then

$$
f_{k}(t, a) \leqslant f_{k}(t, b), \quad \text { for all } k=1, \ldots, N \text {, and a.e. } t \in \mathbb{R} \text {. }
$$

Definition 3.19. A set $E \subset \mathfrak{L} \mathfrak{C}\left(\mathbb{R}^{N}, \mathbb{R}^{N}\right)$ satisfies

$\left(\mathbf{L}_{1}\right)$ if for each $f \in E, j \in \mathbb{N}$ and $k=1, \ldots, N$ there is $u_{f_{k}}^{j} \in L_{l o c}^{1}$ such that, for any $x=\left(x_{1}, \ldots, x_{k}, \ldots, x_{N}\right), \bar{x}^{k}=\left(x_{1} \ldots, \bar{x}_{k}, \ldots, x_{N}\right) \in B_{j} \subset \mathbb{R}^{N}$,

$$
\left|f_{k}(t, x)-f_{k}\left(t, \bar{x}^{k}\right)\right| \leqslant u_{f_{k}}^{j}(t)\left|x_{k}-\bar{x}_{k}\right|, \quad \text { for a.e. } t \in \mathbb{R},
$$

and the set $\left\{u_{f_{k}}^{j} \mid f \in E\right\}$ is $L_{l o c}^{1}$-bounded.

The proofs of the following continuity results are omitted as they can be easily worked out from the ones of Theorems 3.14 and 3.17 .

Proposition 3.20. Consider $E \subset \mathfrak{L} \mathfrak{C}\left(\mathbb{R}^{N}, \mathbb{R}^{N}\right)$ satisfying $\left(\mathbf{L}_{1}\right)$ and such that every element of $E$ satisfies $\left(\mathbf{K}_{1}\right)$. Let $D$ be a countable dense subset of $\mathbb{R}^{N}$.

(i) Let $\left(f_{n}\right)_{n \in \mathbb{N}}$ be a sequence in $E$ converging to $f$ in $\left(\mathfrak{L} \mathfrak{C}\left(\mathbb{R}^{N}, \mathbb{R}^{N}\right), \mathcal{T}_{D}\right)$ and $\left(x_{0, n}\right)_{n \in \mathbb{N}}$ is a sequence converging to $x_{0} \in \mathbb{R}^{N}$, then

$$
x\left(\cdot, f_{n}, x_{0, n}\right) \stackrel{n \rightarrow \infty}{\longrightarrow} x\left(\cdot, f, x_{0}\right)
$$

uniformly in any $\left[T_{1}, T_{2}\right] \subset I_{f, x_{0}}$.

(ii) If $E$ is invariant with respect to the base flow, and $\bar{E}$ denotes the closure of $E$ in $\left(\mathfrak{L C}\left(\mathbb{R}^{N}, \mathbb{R}^{N}\right), \mathcal{T}_{D}\right)$, then

$$
\Phi: \mathcal{U} \subset \mathbb{R} \times \bar{E} \times \mathbb{R}^{N} \rightarrow \bar{E} \times \mathbb{R}^{N}, \quad\left(t, f, x_{0}\right) \mapsto\left(f_{t}, x\left(t, f, x_{0}\right)\right),
$$

is a local monotone continuous skew-product flow.

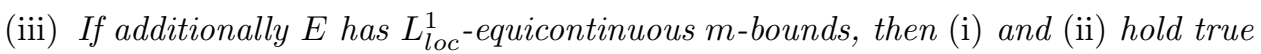
also when the topology $\sigma_{D}$ is employed in place of $\mathcal{T}_{D}$.

Proposition 3.21. Consider $E \subset \mathfrak{L} \mathfrak{C}\left(\mathbb{R}^{N}, \mathbb{R}^{N}\right)$ such that every element of $E$ satisfies $\left(\mathbf{K}_{2}\right)$ and let $D$ be a countable dense subset of $\mathbb{R}^{N}$.

(i) Let $\left(f_{n}\right)_{n \in \mathbb{N}}$ be a sequence in $E$ converging to $f$ in $\left(\mathfrak{L} \mathfrak{C}\left(\mathbb{R}^{N}, \mathbb{R}^{N}\right), \mathcal{T}_{D}\right)$ and $\left(x_{0, n}\right)_{n \in \mathbb{N}}$ is a sequence converging to $x_{0} \in \mathbb{R}^{N}$, then

$$
x\left(\cdot, f_{n}, x_{0, n}\right) \stackrel{n \rightarrow \infty}{\longrightarrow} x\left(\cdot, f, x_{0}\right)
$$

uniformly in any $\left[T_{1}, T_{2}\right] \subset I_{f, x_{0}}$.

(ii) If $E$ is invariant with respect to the base flow, and $\bar{E}$ denotes the closure of $E$ in $\left(\mathfrak{L} \mathfrak{C}\left(\mathbb{R}^{N}, \mathbb{R}^{N}\right), \mathcal{T}_{D}\right)$, then

$$
\Phi: \mathcal{U} \subset \mathbb{R} \times \bar{E} \times \mathbb{R}^{N} \rightarrow \bar{E} \times \mathbb{R}^{N}, \quad\left(t, f, x_{0}\right) \mapsto\left(f_{t}, x\left(t, f, x_{0}\right)\right),
$$

is a local monotone continuous skew-product flow.

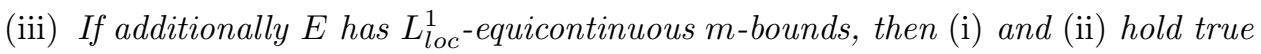
also when the topology $\sigma_{D}$ is employed in place of $\mathcal{T}_{D}$. 


\section{Monotone sublinear CARAThÉOdory SKEW-PROduCT SEMiflows}

Let $E$ be a subset of $\mathfrak{L C}\left(\mathbb{R}^{2 N}, \mathbb{R}^{N}\right)$ invariant with respect to the base flow and closed with respect to a topology $\mathcal{T}$. In the previous section, assuming appropriate conditions on $E$ and $\mathcal{T}$, we obtained several results asserting that the map

$$
\Phi: \mathbb{R}^{+} \times E \times \mathcal{C} \rightarrow E \times \mathcal{C}, \quad(t, f, \phi) \mapsto\left(f_{t}, x_{t}(\cdot, f, \phi)\right)
$$

is a monotone continuous skew-product semiflow. This will be the situation considered throughout the current section. Recall that $\mathcal{C}$ is endowed with a strong partial ordering (3.1) with positive cone $\mathcal{C}^{+}=\{\phi \in \mathcal{C} \mid \phi \geqslant 0\}$.

The following definitions, previously introduced both for deterministic and random monotone semiflows (see [19] and [6]), are of great importance for the long-term behavior of the trajectories. Note that these concepts are associated to the semiflow $\Phi$, although this dependence does not appear explicitly in the definition.

Definition 4.1 (semi-equibrium). A map $a: E \rightarrow \mathcal{C}$ such that $x(t, f, a(f))$ is defined for any $t \geqslant 0$ is

(i) a sub-equilibrium if $a\left(f_{t}\right) \leqslant x_{t}(\cdot, f, a(f))$ for any $f \in E$ and $t \geqslant 0$,

(ii) a super-equilibrium if $x_{t}(\cdot, f, a(f)) \leqslant a\left(f_{t}\right)$ for any $f \in E$ and $t \geqslant 0$,

(iii) an equilibrium if $a\left(f_{t}\right)=x_{t}(\cdot, f, a(f))$ for any $f \in E$ and $t \geqslant 0$.

We will use the term semi-equilibrium to refer either to a super or a sub-equilibrium.

Definition 4.2. A sub-equilibrium (resp. super-equilibrium) $a: E \rightarrow \mathcal{C}$ is lowersemicontinuous (resp. upper-semicontinuous) if for any sequence $\left(f_{n}\right)_{n \in \mathbb{N}}$ converging to some $f$ in $E$ with respect to $\mathcal{T}$ :

(i) $\left\{a\left(f_{n}\right) \mid n \in \mathbb{N}\right\}$ is a relatively compact subset of $\mathcal{C}$, and

(ii) $a(f) \leqslant \phi$ (resp. $\phi \leqslant a(f))$ whenever there is a subsequence $\left(f_{n_{k}}\right)_{k \in \mathbb{N}}$ satisfying $\lim _{k \rightarrow \infty} a\left(f_{n_{k}}\right)=\phi$.

These concepts apply to the case of an equilibrium because it is both a sub- and a super-equilibrium.

The notion of semicontinuous semiequilibrium introduced above will be enough for the purposes of this work. Anyhow, it is easy to check that if $a: E \rightarrow \mathcal{C}$ satisfies Definition 4.2, then it is possible to define an associated multievaluated function $\mathfrak{a}: E \rightarrow \mathcal{C}$ which is upper-semicontinuous in the usual sense (see Aubin and Frankowska [4].

Remark 4.3. Notice that if $a$ is a lower-semicontinuous sub-equilibrium, then the set $\{(f, \phi) \in E \times \mathcal{C} \mid a(f) \leqslant \phi\}$ is a positively invariant closed set. Indeed, the positively invariance follows from Definition 4.1(i). As regards the closeness, consider a sequence $\left(f_{n}, \phi_{n}\right)_{n \in \mathbb{N}}$ converging to some $(f, \phi) \in E \times \mathcal{C}$ and such that for each $n \in \mathbb{N}, a\left(f_{n}\right) \leqslant \phi_{n}$. By the lower-semicontinuity there is a subsequence $\left(f_{n_{k}}\right)_{k \in \mathbb{N}}$ and $\psi \in \mathcal{C}$ such that $\lim _{k \rightarrow \infty} a\left(f_{n_{k}}\right)=\psi$ with $a(f) \leqslant \psi$, and hence, $a(f) \leqslant \psi \leqslant \phi$, as claimed. Similarly, $\{(f, \phi) \in E \times \mathcal{C} \mid b(f) \geqslant \phi\}$ is a positively invariant closed set, provided that $b$ is an upper-semicontinuous super-equilibrium.

Theorem 4.4. Consider $\Phi$ one of the monotone continuous skew-product semiflows given in (4.1). Let $a: E \rightarrow \mathcal{C}$ be a lower-semicontinuous sub-equilibrium and let $b: E \rightarrow \mathcal{C}$ be an upper-semicontinuous super-equilibrium such that $a(f) \leqslant b(f)$ for each $f \in E$, then 
(i) for any $\tau \geqslant 0$ the function

$$
a_{\tau}: E \rightarrow \mathcal{C}, \quad f \mapsto a_{\tau}(f)=x_{\tau}\left(\cdot, f_{-\tau}, a\left(f_{-\tau}\right)\right)
$$

is a lower-semicontinuous sub-equilibrium satisfying

$$
a(f) \leqslant a_{\tau_{1}}(f) \leqslant a_{\tau_{2}}(f) \leqslant b(f) \text { whenever } 0 \leqslant \tau_{1} \leqslant \tau_{2} \text { and } f \in E ;
$$

(ii) the limit $u(f)=\lim _{\tau \rightarrow \infty} a_{\tau}(f)$ exists for any $f \in E$, and $u: E \rightarrow \mathcal{C}$ is a lower-semicontinuous equilibrium;

(iii) for any $\tau>0$ the function

$$
b_{\tau}: E \rightarrow \mathcal{C}, \quad f \mapsto b_{\tau}(f)=x_{\tau}\left(\cdot, f_{-\tau}, b\left(f_{\tau}\right)\right),
$$

is an upper-semicontinuous super-equilibrium satisfying

$$
a(f) \leqslant b_{\tau_{2}}(f) \leqslant b_{\tau_{1}}(f) \leqslant b(f) \text { whenever } 0 \leqslant \tau_{1} \leqslant \tau_{2} \text { and } f \in E ;
$$

(iv) the limit $v(f)=\lim _{\tau \rightarrow \infty} b_{\tau}(f)$ exists for any $f \in E$, and $v: E \rightarrow \mathcal{C}$ is an upper-semicontinuous equilibrium;

(v) for any $f \in E, a(f) \leqslant u(f) \leqslant v(f) \leqslant b(f)$.

Proof. (i) See [6, Proposition 3.4.1] to prove that $a_{\tau}$ is a sub-equilibrium satisfying (4.2) for each fixed $\tau>0$. Next we show that it is lower-semicontinuous. First we check that given a sequence $\left(f_{n}\right)_{n \in \mathbb{N}}$ converging to some $f \in E$ with respect to $\mathcal{T}$, the set $\left\{a_{\tau}\left(f_{n}\right) \mid n \in \mathbb{N}\right\}$ is relatively compact in $\mathcal{C}$, i.e. there is a convergent subsequence. From $\lim _{n \rightarrow \infty}\left(f_{n}\right)_{-\tau}=f_{-\tau}$ and the lower semicontinuity of $a$ we deduce that there is a subsequence $\left(f_{n_{k}}\right)_{k \in \mathbb{N}}$ and $\phi^{\tau} \in \mathcal{C}$ such that

$$
\lim _{k \rightarrow \infty} a\left(\left(f_{n_{k}}\right)_{-\tau}\right)=\phi^{\tau} \quad \text { with } a\left(f_{-\tau}\right) \leqslant \phi^{\tau} .
$$

Then from the continuity of the skew-product semiflow (4.1) we conclude that

$$
a_{\tau}\left(f_{n_{k}}\right)=x_{\tau}\left(\cdot,\left(f_{n_{k}}\right)_{-\tau}, a\left(\left(f_{n_{k}}\right)_{-\tau}\right)\right) \stackrel{k \rightarrow \infty}{\longrightarrow} x_{\tau}\left(\cdot, f_{-\tau}, \phi^{\tau}\right) \text { in } \mathcal{C},
$$

as claimed. Finally, $a_{\tau}(f)=x_{\tau}\left(\cdot, f_{-\tau}, a\left(f_{-\tau}\right)\right) \leqslant x_{\tau}\left(\cdot, f_{-\tau}, \phi^{\tau}\right)$ follows from the monotone character of the skew-product semiflow (4.1), and hence, the lowersemicontinuity of $a_{\tau}$ is obtained.

(ii) First we claim that, for each $f \in E$, the set $\left\{a_{\tau}(f) \mid \tau \geqslant 1\right\} \subset \mathcal{C}$ is relatively compact. The boundedness follows from (4.2), and the equicontinuity from the cocycle property

$$
a_{\tau}(f)=x_{\tau}\left(\cdot, f_{-\tau}, a\left(f_{-\tau}\right)\right)=x_{1}\left(\cdot, f_{-1}, x_{\tau-1}\left(\cdot, f_{-\tau}, a\left(f_{-\tau}\right)\right)\right)=x_{1}\left(\cdot, f_{-1}, a_{\tau-1}\left(f_{-1}\right)\right)
$$

and the $m$-bound for $f_{-1}$ because $\left\{a_{\tau-1}\left(f_{-1}\right) \mid \tau \geqslant 1\right\}$ is also bounded. As a consequence, together with the monotonicity property (4.2), we deduce that there exist a unique $u(f)=\lim _{\tau \rightarrow \infty} a_{\tau}(f)=\sup _{\tau \geqslant 0} a_{\tau}(f)$ in $\mathcal{C}$, as stated. In order to check that $u$ is an equilibrium, notice that

$$
\begin{aligned}
x_{t}(\cdot, f, u(f)) & =\lim _{\tau \rightarrow \infty} x_{t}\left(\cdot, f, a_{\tau}(f)\right)=\lim _{\tau \rightarrow \infty} x_{t}\left(\cdot, f, x_{\tau}\left(\cdot, f_{-\tau}, a\left(f_{-\tau}\right)\right)\right) \\
& =\lim _{\tau \rightarrow \infty} x_{t+\tau}\left(\cdot, f_{-\tau}, a\left(f_{-\tau}\right)\right)=\lim _{\tau \rightarrow \infty} a_{t+\tau}\left(f_{t}\right)=u\left(f_{t}\right)
\end{aligned}
$$

whenever $t \geqslant 0$ and $f \in E$. Finally, we prove that it is lower-semicontinuous. Let $\left(f_{n}\right)_{n \in \mathbb{N}}$ be a sequence converging to some $f \in E$ with respect to $\mathcal{T}$. We check that $\left\{u\left(f_{n}\right) \mid n \in \mathbb{N}\right\}$ is relatively compact. The boundedness follows from (4.2), 
the lower-semicontinuity of $a$ and the upper-semicontinuity of $b$. Next notice that, since $u$ is an equilibrium, we have

$$
u\left(f_{n}\right)=x_{1}\left(\cdot,\left(f_{n}\right)_{-1}, u\left(\left(f_{n}\right)_{-1}\right)\right)=x\left(1+\cdot,\left(f_{n}\right)_{-1}, u\left(\left(f_{n}\right)_{-1}\right)\right) \text { for each } n \in \mathbb{N},
$$

and then, if we are in one of the cases for which $E$ has $L_{l o c}^{1}$-equicontinuous $m$ bounds, the equicontinuity follows from the boundedness of the set of initial data $\left\{u\left(\left(f_{n}\right)_{-1}\right) \mid n \in \mathbb{N}\right\}$. When the $L_{l o c}^{1}$-equicontinuity of the $m$-bounds is not verified, that is, Theorem 3.14[(i)-(ii)] and Theorem 3.17[(i)-(ii)], the equicontinuity follows from similar arguments to the ones applied to check the equicontinuity of the sequence $\left(z_{n}\right)_{n \in \mathbb{N}}$ in these theorems, see inequalities (3.11) and (3.14), respectively. Therefore, $\lim _{k \rightarrow \infty} u\left(f_{n_{k}}\right)=\phi$ for some $\phi$, and from the lower-semicontinuity of $a_{\tau}$, up to a subsequence, there is a $\psi^{\tau} \in \mathcal{C}$ with

$$
a_{\tau}(f) \leqslant \psi^{\tau}=\lim _{k \rightarrow \infty} a_{\tau}\left(f_{n_{k}}\right) \quad \text { for each } \tau \geqslant 0 .
$$

From $a_{\tau}\left(f_{n_{k}}\right) \leqslant u\left(f_{n_{k}}\right)$ we deduce that $\psi^{\tau} \leqslant \phi$, and thus, $u(f)=\sup _{\tau \geqslant 0} a_{\tau}(f) \leqslant \phi$, which finish the proof of the lower-semicontinuity.

(iii) and (iv) can be proved reasoning as for (i) and (ii) respectively, but inverting the appropriate inequalities accordingly. (v) follows from (4.2) and (4.3).

Next we introduce the concepts of sublinear functions and sublinear skew-product semiflows in our context.

Definition 4.5. A function $f \in \mathfrak{L} \mathfrak{C}\left(\mathbb{R}^{2 N}, \mathbb{R}^{N}\right)$ is said to be sublinear if

(S) for each $x, y \in \mathbb{R}^{N}, x, y \geqslant 0$, and each $\lambda \in[0,1]$

$$
f(t, \lambda x, \lambda y) \geqslant \lambda f(t, x, y) \quad \text { for a.e. } t \in \mathbb{R} .
$$

A subset $E \subset \mathfrak{L} \mathfrak{C}\left(\mathbb{R}^{2 N}, \mathbb{R}^{N}\right)$ is said to be sublinear or to satisfy (S) if all its elements are sublinear.

Definition 4.6. The skew-product semiflow (4.1) is said to be sublinear if for each function $f \in E$

$$
x_{t}(\cdot, f, \lambda \phi) \geqslant \lambda x_{t}(\cdot, f, \phi) \quad \text { whenever } t>0, \lambda \in[0,1] \text { and } \phi \geqslant 0 .
$$

A function $f \in \mathfrak{L} \mathfrak{C}\left(\mathbb{R}^{2 N}, \mathbb{R}^{N}\right)$ is said to be a point of strong sublinearity for $\Phi$ if

$$
x_{t}(\cdot, f, \lambda \phi) \gg \lambda x_{t}(\cdot, f, \phi) \quad \text { whenever } t>1, \lambda \in(0,1) \text { and } \phi \gg 0 .
$$

The next technical lemma allows us to pass from $(\mathbf{S})$, formulated pointwise, to a similar condition involving continuous functions. We omit the proof, analogous to the one of Lemma 3.3.

Lemma 4.7. If $f \in \mathfrak{L} \mathfrak{C}\left(\mathbb{R}^{2 N}, \mathbb{R}^{N}\right)$ satisfies $(\mathbf{S})$, then for each $\lambda \in[0,1]$, any interval $I \subset \mathbb{R}$ and any functions $x, y \in \mathcal{C}\left(I, \mathbb{R}^{N}\right)$, with $x, y \geqslant 0$ one has that

$$
f(t, \lambda x(t), \lambda y(t)) \geqslant \lambda f(t, x(t), y(t)), \quad \text { for a.e. } t \in I .
$$

The sublinear character of the skew-product semiflow can be deduced from the sublinearity of all the functions in $E$ as shown in the next result.

Proposition 4.8. Consider $\Phi$ one of the monotone continuous skew-product semiflows given in (4.1) and assume that E satisfies property $(\mathbf{S})$. Then, (4.1) preserves the positive cone and induces a monotone and sublinear skew-product semiflow

$$
\Phi: \mathbb{R}^{+} \times E \times \mathcal{C}^{+} \rightarrow E \times \mathcal{C}^{+}, \quad(t, f, \phi) \mapsto\left(f_{t}, x_{t}(\cdot, f, \phi)\right) .
$$


Proof. The case $\lambda=1$ is trivial so let us fix $f \in E, \lambda \in[0,1)$ and $\phi \gg 0$. We firstly show that $x(t, f, \lambda \phi) \geqslant \lambda x(t, f, \phi)$ for $t \in[0,1]$. Take

$$
t_{1}=\sup \{\tau \in[0,1] \mid x(t, f, \phi) \geqslant 0 \text { for all } t \in[0, \tau]\} .
$$

It is obvious that $t_{1}>0$. For each $t \in\left[0, t_{1}\right]$ denote by $v(t)=\lambda x(t, f, \phi)$ and $w(t)=x(t, f, \lambda \phi)$ and notice that $w^{\prime}(t)=g(t, w(t))$ where $g: \mathbb{R} \times \mathbb{R}^{N} \rightarrow \mathbb{R}^{N}$, $(t, y) \mapsto f(t, y, \lambda \phi(t-1))$. Hence, from Lemma 4.7

$$
v^{\prime}(t)-g(t, v(t))=\lambda f(t, x(t, f, \phi), \phi(t-1))-g(t, v(t)) \leqslant 0=w^{\prime}(t)-g(t, w(t))
$$

a.e. in $\left[0, t_{1}\right]$, which together with $v(0)=\lambda \phi(0)=w(0)$ and Theorem 2 of [28] provides $v(t) \leqslant w(t)$ for $t \in\left[0, t_{1}\right]$. Next, from Lemma 3.9 and $\phi(0) \gg \lambda \phi(0)$ we deduce that $x\left(t_{1}, f, \phi\right) \gg x\left(t_{1}, f, \lambda \phi\right)$, which along with $x\left(t_{1}, f, \lambda \phi\right) \geqslant \lambda x\left(t_{1}, f, \phi\right) \geqslant$ 0 shows that it must be $t_{1}=1$, and we obtain the claimed inequality. In a recursive way we show the case $t>1$, and the sublinearity of the semiflow follows. Finally, notice that from the monotonicity, if $\phi \geqslant 0$ we deduce that $x_{t}(\cdot, f, \phi) \geqslant x_{t}(\cdot, f, 0)$ and from (4.4) with $\lambda=0$ the invariance of the positive cone $\mathcal{C}^{+}$is obtained.

As a final result of this section, we show that under the assumptions of Theorem 4.4 with the addition of sublinearity for the monotone continuous skew-product semiflows (4.6), further important dynamic information can be drawn upon the system. Indeed, one can single out two invariant subsets of $E$ where either the semicontinuous equilibria provided by Theorem 4.4 are in fact continuous and coincide, or where they determine the forward long-term behavior of the solutions. In order to state and prove the result, let us firstly recall the definition of part metric.

Definition 4.9 (Part metric). Consider the equivalence relation on $\mathcal{C}^{+}$defined by $x \sim y$ if and only if there is $\alpha>0$ such that $\alpha^{-1} x \leqslant y \leqslant \alpha x$. The classes of equivalence in $\mathcal{C}^{+}$are called the parts of $\mathcal{C}^{+}$.

If $C$ is a part of $\mathcal{C}^{+}$, then

$$
p(x, y):=\inf \left\{\log \alpha \mid \alpha^{-1} x \leqslant y \leqslant \alpha x\right\}, \quad x, y \in C,
$$

defines a metric on $C$ called the part metric of $C$.

It is easy to check that $\operatorname{Int} \mathcal{C}^{+}=\{\phi \in \mathcal{C} \mid \phi \gg 0\}$ is a part of $\mathcal{C}^{+}$.

Theorem 4.10. Let $\Phi$ be one of the monotone and sublinear continuous skewproduct semiflows (4.6) induced by (4.1) in the sublinear case. Let $u, v: E \rightarrow \operatorname{Int} \mathcal{C}^{+}$ be the semicontinuous equilibria provided by Theorem 4.4 from the semiequilibria $a, b: E \rightarrow \operatorname{Int} \mathcal{C}^{+}$, and consider the sets

$$
\begin{aligned}
& E_{-}:=\left\{\begin{array}{l|l}
f \in E & \begin{array}{l}
\text { there is a sequence } t_{n} \downarrow-\infty \text { and } \\
\text { a point of strong sublinearity } g \in E
\end{array} \quad \text { with } \lim _{n \rightarrow \infty} f_{t_{n}}=g
\end{array}\right\}, \\
& E_{+}:=\left\{\begin{array}{l|l}
f \in E & \begin{array}{l}
\text { there is a sequence } t_{n} \uparrow+\infty \text { and } \\
\text { a point of strong sublinearity } g \in E
\end{array} \text { with } \lim _{n \rightarrow \infty} f_{t_{n}}=g
\end{array}\right\} .
\end{aligned}
$$

Then,

(i) $E_{-}$and $E_{+}$are invariant.

(ii) Each function $f \in E_{-}$is a continuity point for $u$ and $v$ and $u(f)=v(f)$. In particular, $u$ and $v$ are continuous in $E_{-}$.

(iii) For each $f \in E_{+}$and $\phi \in \operatorname{Int} \mathcal{C}^{+}$

$$
\lim _{t \rightarrow \infty} p\left(u\left(f_{t}\right), x_{t}(\cdot, f, \phi)\right)=\lim _{t \rightarrow \infty} p\left(v\left(f_{t}\right), x_{t}(\cdot, f, \phi)\right)=0 .
$$


In particular, if $\left(t_{n}\right)_{n \in \mathbb{N}}$ is such that $t_{n} \uparrow \infty$ and $\lim _{n \rightarrow \infty} f_{t_{n}}=g \in E$, then

$$
\lim _{n \rightarrow \infty}\left\|u\left(f_{t_{n}}\right)-x_{t_{n}}(\cdot, f, \phi)\right\|_{\mathcal{C}}=\lim _{n \rightarrow \infty}\left\|v\left(f_{t_{n}}\right)-x_{t_{n}}(\cdot, f, \phi)\right\|_{\mathcal{C}}=0 .
$$

Proof. (i) Let $f \in E_{-}$and $s \in \mathbb{R}$. If we consider the sequence $\left(t_{n}-s\right)_{n \in \mathbb{N}}$, it is immediate to check that $\lim _{n \rightarrow \infty}\left(t_{n}-s\right)=-\infty$ and $\lim _{n \rightarrow \infty}\left(f_{s}\right)_{t_{n}-s}=g$, so that $f_{s} \in E_{-}$.

(ii) Let $f \in E_{-}$. Then, there exists a sequence $t_{n} \downarrow-\infty$, which can be assumed to satisfy $t_{n}<0$ and $t_{n-1}-t_{n}>2$ for each $n \in \mathbb{N}$, and a point of strong sublinearity $g \in E$ such that $\lim _{n \rightarrow \infty} f_{t_{n}}=g$. First we check that $u(f)=v(f)$. Since $u$ is a lower-semicontinuos equilibrium and $v$ an upper-semicontinuos equilibrium, up to a subsequence, there are $\widetilde{u}$ and $\widetilde{v} \in E$ such that

$$
0 \ll u(g) \leqslant \widetilde{u}=\lim _{n \rightarrow \infty} u\left(f_{t_{n}}\right) \leqslant \lim _{n \rightarrow \infty} v\left(f_{t_{n}}\right)=\widetilde{v} \leqslant v(g) .
$$

First notice that since $u$ and $v$ are equilibria, and the part metric is decreasing along the trajectories because of the sublinearity of the semiflow (see [6, Lemma 4.2.1(i)]), we deduce that

$$
p\left(u\left(f_{t}\right), v\left(f_{t}\right)\right) \geqslant p\left(u\left(f_{s}\right), v\left(f_{s}\right)\right) \quad \text { whenever } t \leqslant s .
$$

Next we claim that $p(\widetilde{u}, \widetilde{v})=0$. Otherwise, since $g$ is a point of strong sublinearity (see (4.5)), the contracting property under the part metric for the trajectory (see [6, Lemma 4.2.1(ii)]), the continuity of the semiflow, the inequalities $t_{n}+2<t_{n-1}$ for each $n \in \mathbb{N}$ and (4.9) provide

$$
\begin{aligned}
p(\widetilde{u}, \widetilde{v}) & \left.>p\left(x_{2}(\cdot, g, \widetilde{u})\right), x_{2}(\cdot, g, \widetilde{v})\right)=\lim _{n \rightarrow \infty} p\left(x_{2}\left(\cdot, f_{t_{n}}, u\left(f_{t_{n}}\right)\right), x_{2}\left(\cdot, f_{t_{n}}, v\left(f_{t_{n}}\right)\right)\right) \\
& =\lim _{n \rightarrow \infty} p\left(u\left(f_{t_{n}+2}\right), v\left(f_{t_{n}+2}\right)\right) \geqslant \lim _{n \rightarrow \infty} p\left(u\left(f_{t_{n-1}}\right), v\left(f_{t_{n-1}}\right)\right)=p(\widetilde{u}, \widetilde{v}),
\end{aligned}
$$

a contradiction, and $p(\widetilde{u}, \widetilde{v})=0$, as claimed. Finally, again from (4.9) and $t_{n}<0$ we deduce that $0 \leqslant p(u(f), v(f)) \leqslant p\left(u\left(f_{t_{n}}\right), v\left(f_{t_{n}}\right)\right)$, which as $n$ goes to $\infty$ yields $0 \leqslant p(u(f), v(f)) \leqslant p(\widetilde{u}, \widetilde{v})=0$, that is, $u(f)=v(f)$, as stated.

Now we check that each $f \in E_{-}$is a continuity point for $u$ and $v$. Again, from the lower- and upper-semicontinuity of the equilibria and Theorem 4.4(v), we deduce that for each sequence $\left(f_{n}\right)_{n \in \mathbb{N}}$ converging to $f$, there is a subsequence $\left(f_{n_{k}}\right)_{k \in \mathbb{N}}$ such that

$$
u(f) \leqslant \lim _{k \rightarrow \infty} u\left(f_{n_{k}}\right) \leqslant \lim _{k \rightarrow \infty} v\left(f_{n_{k}}\right) \leqslant v(f) .
$$

Thus, from $u(f)=v(f)$ we conclude that $\lim _{k \rightarrow \infty} u\left(f_{n_{k}}\right)=\lim _{k \rightarrow \infty} v\left(f_{n_{k}}\right)=u(f)=v(f)$, and the continuity of $u$ and $v$ in $E_{-}$is obtained.

(iii) We will show the results for $u$ because the corresponding ones for $v$ are analogous. Let $f \in E_{+}$and $\phi \gg 0$. As in (4.9), from the sublinear character of the semiflow, the function $P(t)=p\left(u\left(f_{t}\right), x_{t}(\cdot, f, \phi)\right)$ is positive and decreasing in $t>0$, that is, $P(t) \geqslant P(s) \geqslant 0$ whenever $0<t \leqslant s$, and hence there exists the limit as $t \uparrow \infty$. We check that it is 0 as claimed.

Since $f \in E_{+}$, there is a sequence $t_{n} \uparrow \infty$ which can be assumed to satisfy $t_{n}>0$ and $t_{n+1}-t_{n}>2$ for each $n \in \mathbb{N}$, and a point of strong sublinearity $g \in E$ such that $\lim _{n \rightarrow \infty} f_{t_{n}}=g$. Again, from the lower-semicontinuity of $u$ there is a subsequence, we will take the whole one, and $\widetilde{u} \in E$ satisfying $0 \ll u(g) \leqslant \widetilde{u}=\lim _{n \rightarrow \infty} u\left(f_{t_{n}}\right)$. 
We assume that $p(u(f), \phi)=P(0)>0$, otherwise $P$ would vanish on $\mathbb{R}^{+}$and the claim is trivially true. Then, there is an $\alpha>1$ such that $\alpha^{-1} u(f) \leqslant \phi \leqslant \alpha u(f)$, and the monotonicity of the semiflow provides

$$
x_{t}\left(\cdot, f, \alpha^{-1} u(f)\right) \leqslant x_{t}(\cdot, f, \phi) \leqslant x_{t}(\cdot, f, \alpha u(f)) .
$$

Moreover, from the sublinearity of the semiflow, since $\alpha^{-1}<1$ we deduce that

$$
\begin{aligned}
& \alpha^{-1} u\left(f_{t}\right)=\alpha^{-1} x_{t}(\cdot, f, u(f)) \leqslant x_{t}\left(\cdot, f, \alpha^{-1} u(f)\right) \quad \text { and } \\
& x_{t}(\cdot, f, \alpha u(f)) \leqslant \alpha x_{t}(\cdot, f, u(f))=\alpha u\left(f_{t}\right),
\end{aligned}
$$

which, together with (4.10) and evaluating at $t_{n}$, show that $\left\{x_{t_{n}}(\cdot, f, \phi) \mid n \in \mathbb{N}\right\}$ is bounded because

$$
\alpha^{-1} u\left(f_{t_{n}}\right) \leqslant x_{t_{n}}(\cdot, f, \phi) \leqslant \alpha u\left(f_{t_{n}}\right)
$$

and $u\left(f_{t_{n}}\right) \rightarrow \widetilde{u}$ as $n \uparrow \infty$. We omit the proof of the equicontinuity because follows the same arguments of Theorem 4.4 for proving that $\left\{u\left(f_{n}\right) \mid n \in \mathbb{N}\right\}$ was equicontinuous. Consequently, $\left\{x_{t_{n}}(\cdot, f, \phi) \mid n \in \mathbb{N}\right\}$ is a relatively compact set and there is a convergent subsequence. For simplicity of notation we will assume that the sequence itself converges, and let denote the limit by $\widetilde{x}$. We claim that $p(\widetilde{u}, \widetilde{x})=0$. Otherwise, the same arguments of (ii) together with $t_{n}+2<t_{n+1}$ provide now the chain of inequalities

$$
\begin{aligned}
p(\widetilde{u}, \widetilde{x}) & >p\left(x_{2}(\cdot, g, \widetilde{u}), x_{2}(\cdot, g, \widetilde{x})\right)=\lim _{n \rightarrow \infty} p\left(x_{2}\left(\cdot, f_{t_{n}}, u\left(f_{t_{n}}\right)\right), x_{2}\left(\cdot, f_{t_{n}}, x_{t_{n}}(\cdot, f, \phi)\right)\right. \\
& =\lim _{n \rightarrow \infty} P\left(t_{n}+2\right) \geqslant \lim _{n \rightarrow \infty} P\left(t_{n+1}\right)=p(\widetilde{u}, \widetilde{x}),
\end{aligned}
$$

and hence, $p(\widetilde{u}, \widetilde{x})=0$ and $\lim _{t \rightarrow \infty} P(t)=0$, as stated. Finally, (4.8) follows from (4.7), the relation (see Krause and Nussbaum [11, Lemma 2.3(ii)])

$$
\|x-y\| \leqslant\left(2 e^{p(x, y)}-e^{-p(x, y)}-1\right) \min (\|x\|,\|y\|),
$$

and the boundedness of $\left\{u\left(f_{t_{n}}\right) \mid n \in \mathbb{N}\right\}$ and $\left\{x_{t_{n}}(\cdot, f, \phi) \mid n \in \mathbb{N}\right\}$.

\section{Some APplications}

In this section, we will show the importance of the applications of our theory to the study of nonautonomous Carathéodory ordinary and delay cooperative systems of equations arising in several applied sciences.

5.1. Scalar model for population dynamics. The use of scalar differential equations with constant delay to model the dynamics of a population is extensive and effective (see Brauer and Chávez [5] and Smith [25]). We will study a model in population biology given by scalar delay Carathéodory equations of the form

$$
x^{\prime}(t)=-\alpha(t) x(t)+h(t, x(t-1)),
$$

which will be compared with the linear Carathéodory ones

$$
y^{\prime}(t)=-\alpha(t) y(t)+\beta(t) y(t-1)+\gamma(t) .
$$

Equations like (5.1) include for example Nicholson's model, Mackey-Glass's model and similar, which have been studied in depth in mathematical biology. First we state the assumptions to be considered for the families of equations (5.1) and (5.2). 
(A1) $E_{1}$ is a closed invariant and bounded subset of functions $\alpha \in L_{l o c}^{1}$ such that $\alpha(t) \geqslant 0$ for a.e. $t \in \mathbb{R}$ and the null function $\alpha=0$ does not belong to $E_{1}$. Let $D$ be a countable dense subset of $\mathbb{R}$ and consider the subset of $\mathfrak{L} \mathfrak{C}$ given by

$$
E_{2}=\left\{h \in \mathfrak{L} \mathfrak{C} \mid \begin{array}{l}
h \text { satisfies }\left(\mathbf{K}_{2}\right), h_{0}(t):=h(t, 0) \in E_{1} \\
\text { and } h(t, y)=h(t, 0) \text { for } y \leqslant 0
\end{array}\right\} .
$$

Notice that $E_{2}$ is invariant and closed with respect to the topology $\mathcal{T}_{D}$.

(A2) $E$ is a subset of

$$
\left\{\begin{array}{l|l}
f=(h, \alpha, \beta, \gamma) & \begin{array}{l}
h \in E_{2}, \alpha, \beta, \gamma \in E_{1} \text { and } \\
h(t, y) \leqslant \beta(t) y+\gamma(t), \forall y \in \mathbb{R}, \text { a.e. } t \in \mathbb{R}
\end{array}
\end{array}\right\}
$$

which is invariant and closed for the product topology.

(A3) There are positive constants $K, \delta>0$ such that for each $f=(h, \alpha, \beta, \gamma) \in E$

$$
\|T(t, f)\| \leqslant K e^{-\delta t} \quad \text { for each } t>0,
$$

where $T(t, f)$ is the evolution operator on $C([-1,0], \mathbb{R})$ for $(5.3)$, that is, $T(t, f) \phi=x_{t}(\cdot, f, \phi)$ is the unique solution of the linear equation

$$
z^{\prime}(t)=-\alpha(t) z(t)+\beta(t) z(t-1)
$$

with initial data $\phi \in C([-1,0], \mathbb{R})$.

Under these assumptions, the functions defining equations (5.1) and (5.2), that is, $g(t, x, y)=\alpha(t) x+h(t, y)$ and $\widehat{g}(t, x, y)=\alpha(t) x+\beta(t) y+\gamma(t)$, satisfy conditions $\left(\mathbf{K}_{x}\right),\left(\mathbf{K}_{y}\right)$ and $\left(\mathbf{L}_{x}\right)$. Therefore, from Theorem 3.7 and Theorem 3.14 we deduce that the skew-product semiflows:

$$
\begin{aligned}
& \Phi: \mathbb{R}^{+} \times E \times \mathcal{C}^{+} \rightarrow E \times \mathcal{C}^{+}, \quad(t, f, \phi) \mapsto\left(f_{t}, x_{t}(\cdot, f, \phi)\right), \\
& \Psi: \mathbb{R}^{+} \times E \times \mathcal{C}^{+} \rightarrow E \times \mathcal{C}^{+}, \quad(t, f, \phi) \mapsto\left(f_{t}, y_{t}(\cdot, f, \phi)\right),
\end{aligned}
$$

are monotone and continuous for the above product topology.

Remark 5.1. Let $f=(h, \alpha, \beta, \gamma) \in E$. We denote by $U_{f}(t, s)$ the so called fundamental matrix of (5.3) (scalar in this case, see [8, Theorem 2.1]), that is,

$$
\begin{aligned}
& \frac{d}{d t} U_{f}(t, s)=-\alpha(t) U_{f}(t, s)+\beta(t) U_{f}(t-1, s), \text { if } t \geqslant s \text { and a.e. in } s \text { and } t . \\
& U_{f}(s, s)=1 \text { and } U(t, s)=0 \text { for } s-1 \leqslant t<s .
\end{aligned}
$$

Moreover, it is also assumed that $U_{f}(t, s)=0$ whenever $t<s$. Notice that for each $f=(h, \alpha, \beta, \gamma) \in E$ and assumption (A3) we have

$$
\left|U_{f}(t, s)\right| \leqslant K e^{-\delta(t-s)} \quad \text { whenever } t \geqslant s .
$$

The reason is that if we change the initial condition (5.6) to the constant function 1 on $[s-1, s]$, and we denote this solution by $z(t, f, s, 1)$, it is easy to check that

$$
U_{f}(t, s) \leqslant z(t, f, s, 1)=z\left(t-s, f_{s}, 1\right),
$$

and (A3) proves the claim.

Proposition 5.2. Under assumptions (A1)-(A3) and the notation of Remark 5.1,

(i) the function $\widetilde{b}: E \rightarrow \mathbb{R}, f \mapsto \widetilde{b}(f)=\int_{-\infty}^{0} U_{f}(0, s) \gamma(s) d s$ is well defined and continuous, 
(ii) the function $b: E \rightarrow \mathcal{C}^{+}, f \mapsto b(f)$, defined as $b(f)(u)=\widetilde{b}\left(f_{u}\right)$ for each $u \in[-1,0]$, is a continuous equilibrium for (5.5) and a continuous superequilibrium for (5.4),

(iii) the function $\widetilde{a}: E \rightarrow \mathbb{R}, f \mapsto \widetilde{a}(f)=\int_{-\infty}^{0} \exp \left(-\int_{s}^{0} \alpha(r) d r\right) h_{0}(s) d s$, with $h_{0}$ defined in (A1), is well defined and continuous,

(iv) the function $a: E \rightarrow \mathcal{C}^{+}, f \mapsto a(f)$, defined as $a(f)(u)=\widetilde{a}\left(f_{u}\right)$ for each $u \in[-1,0]$, is a continuous sub-equilibrium for (5.4), and

they satisfy $0 \ll a(f) \leqslant b(f)$ for each $f \in E$.

Proof. (i) Notice that (A1) implies that the set $\left\{\gamma_{t} \mid \gamma \in E_{1}, t \in \mathbb{R}\right\}$ is $L_{l o c}^{1}$-bounded, so there is a constant $C \geqslant 0$ such that

$$
\int_{t}^{t+1} \gamma(s) d s \leqslant C \quad \text { for each } t \in \mathbb{R} \text { and } \gamma \in E_{1} .
$$

From this together with (5.7) we deduce that for each $f=(h, \alpha, \beta, \gamma) \in E$

$$
\left|\int_{-\infty}^{0} U_{f}(0, s) \gamma(s) d s\right| \leqslant \sum_{j=1}^{\infty} \int_{-j}^{-j+1} K e^{\delta s} \gamma(s) d s \leqslant \sum_{j=1}^{\infty} K C e^{(-j+1) \delta}=K C \frac{e^{\delta}}{e^{\delta}-1},
$$

so that the integral is well defined, and since the bound is independent of $f$, we deduce that given $\varepsilon>0$ there is a $\tau_{\varepsilon}>0$ such that

$$
\left|\int_{-\infty}^{-\tau_{\varepsilon}} U_{f}(0, s) \gamma(s) d s\right|<\varepsilon \quad \text { for each } f=(h, \alpha, \beta, \gamma) \in E .
$$

Before proving the continuity of $\widetilde{b}$, we will study some properties of $U_{f}(0, s)$. From (5.6), if $s \leqslant t \leqslant s+1$ we deduce that $U_{f}(t, s)$ is the solution of the Carathéodory ordinary differential problem $z^{\prime}(t)=-\alpha(t) z(t), z(s)=1$, i.e.

$$
U_{f}(t, s)=\exp \left(-\int_{s}^{t} \alpha(r) d r\right) \quad \text { whenever } s \leqslant t \leqslant s+1 .
$$

As in Remark 5.1, we denote by $z(t, f, \phi)$ the solution of

$$
\left\{\begin{array}{l}
z^{\prime}(t)=-\alpha(t) z(t)+\beta(t) z(t-1), \\
z(t)=\phi(t), \quad t \in[-1,0],
\end{array} \quad \text { for } f=(h, \alpha, \beta, \gamma) \in E, \text { and } \phi \in \mathcal{C}([-1,0]) .\right.
$$

Since the cocycle property for $z_{t}(\cdot, f, \phi)$ can be also applied to the non-continuous initial data $\phi_{0}:[-1,0] \rightarrow \mathbb{R}$, defined as $\phi_{0}(t)=0$ if $t \in[-1,0)$ and $\phi_{0}(0)=1$, we deduce that if $t \geqslant s+1$

$$
U_{f}(t, s)=z\left(t-s, f_{s}, \phi_{0}\right)=z\left(t-s-1, f_{s+1}, z_{1}\left(\cdot, f_{s}, \phi_{0}\right)\right),
$$

where now, from the definition of $\phi_{0}$, we have that $z\left(1+u, f_{s}, \phi_{0}\right)$ is the solution of the Carathéodory ordinary differential equation $z^{\prime}(t)=-\alpha(t+s) z(t)$ with initial data $z(0)=1$, and hence,

$$
\phi\left(f_{s}\right)(\tau):=z_{1}\left(\tau, f_{s}, \phi_{0}\right)=\exp \left(-\int_{0}^{1+\tau} \alpha(s+r) d r\right), \tau \in[-1,0] .
$$

Therefore, from (5.10), (5.11) and (5.12) we deduce that

$$
U_{f}(0, s)= \begin{cases}\exp \left(-\int_{s}^{0} \alpha(r) d r\right) & \text { if } s \in[-1,0] \\ z\left(-s-1, f_{s+1}, \phi\left(f_{s}\right)\right) & \text { if } s \leqslant-1\end{cases}
$$


From Theorem 3.14, the map $\left.\mathbb{R}^{-} \times E \times \mathcal{C}([-1,0]) \rightarrow \mathbb{R},(s, g, \phi) \mapsto z(-s, g, \phi)\right)$ is continuous, which, together with (5.13) and the composition with some continuous functions, show the continuity of

$$
\mathbb{R}^{-} \times E \rightarrow \mathbb{R},(s, f) \mapsto U_{f}(0, s) .
$$

To check the continuity of $\widetilde{b}$, we consider a sequence $\left(f_{n}\right)_{n \in \mathbb{N}}=\left(h_{n}, \alpha_{n}, \beta_{n}, \gamma_{n}\right)_{n \in \mathbb{N}}$ on $E$ converging to some $f=(h, \alpha, \beta, \gamma) \in E$ for the product topology and we will check that $\widetilde{b}\left(f_{n}\right)$ tends to $\widetilde{b}(f)$ as $n \uparrow \infty$. Given $\varepsilon>0$, we consider $\tau_{\varepsilon}$ satisfying (5.9) and we denote by $I_{\varepsilon}=\sup _{\gamma \in E_{1}} \int_{-\tau_{\varepsilon}}^{0} \gamma(s) d s$. From the definition of $E_{1}$ given on (A1) we deduce that $0<I_{\varepsilon}<\infty$. Moreover, notice that the restriction of (5.14) to the compact subset $\left[-\tau_{\varepsilon}, 0\right] \times\left(\left\{f_{n} \mid n \in \mathbb{N}\right\} \cup\{f\}\right) \subset \mathbb{R}^{-} \times E$ provides

$$
\lim _{n \rightarrow \infty} U_{f_{n}}(0, s)=U_{f}(0, s) \quad \text { uniformly for } s \in\left[-\tau_{\varepsilon}, 0\right] \text {. }
$$

In addition, $\lim _{n \rightarrow \infty} \gamma_{n}=\gamma$ in $L_{l o c}^{1}$, so that there is an $n_{0}$ such that for each $n \geqslant n_{0}$

$$
\sup _{s \in\left[-\tau_{\varepsilon}, 0\right]}\left|U_{f_{n}}(0, s)-U_{f}(0, s)\right|<\frac{\varepsilon}{I_{\varepsilon}} \quad \text { and } \quad \int_{-\tau_{\varepsilon}}^{0}\left|\gamma_{n}(s)-\gamma(s)\right|<\varepsilon .
$$

As a consequence, together with (5.7), (5.8), (5.9) and

$U_{f_{n}}(0, s) \gamma_{n}(s)-U_{f}(0, s) \gamma(s)=\left(U_{f_{n}}(0, s)-U_{f}(0, s)\right) \gamma_{n}(s)+U_{f}(0, s)\left(\gamma_{n}(s)-\gamma(s)\right)$, we obtain that for each $n \geqslant n_{0}$

$$
\begin{aligned}
\left|\widetilde{b}\left(f_{n}\right)-\widetilde{b}(f)\right| & \leqslant 2 \varepsilon+\int_{-\tau_{\varepsilon}}^{0}\left|U_{f_{n}}(0, s) \gamma_{n}(s)-U_{f}(0, s) \gamma(s)\right| d s \\
& \leqslant 2 \varepsilon+\frac{\varepsilon}{I_{\varepsilon}} \int_{-\tau_{\varepsilon}}^{0}\left|\gamma_{n}(s)\right| d s+\varepsilon \sup _{s \in \mathbb{R}^{-}}\left|U_{f}(0, s)\right| \leqslant \varepsilon(3+K),
\end{aligned}
$$

which proves our claim, and finishes the proof of (i).

(ii) First notice that, from the variation of constant formula, the solution of

$$
\left\{\begin{array}{l}
y^{\prime}(t)=-\alpha(t) y(t)+\beta(t) y(t-1)+\gamma(t), \\
y(t)=0, \quad t \in[s-1, s]
\end{array}\right.
$$

for $f=(h, \alpha, \beta, \gamma) \in E$ is given by $\int_{s}^{t} U_{f}(t, s) \gamma(s) d s$ for each $t \geqslant s$, and, as a consequence of (5.7), it can be shown that

$$
y(t, f, b(f)))=\int_{-\infty}^{t} U_{f}(t, s) \gamma(s) d s
$$

is a bounded and globally defined solution of (5.2) such that $y(s, f, b(f))=b(f)(s)$ for each $s \in[-1,0]$.

Next, as in (5.11), from $U_{f}(t, s)=z\left(t-s, f_{s}, \phi_{0}\right)$ we deduce that

$$
U_{f_{t}}(0, s-t)=z\left(t-s,\left(f_{t}\right)_{s-t}, \phi_{0}\right)=z\left(t-s, f_{s}, \phi_{0}\right)=U_{f}(t, s) .
$$

Therefore,

$$
\tilde{b}\left(f_{t}\right)=\int_{-\infty}^{0} U_{f_{t}}(0, s) \gamma_{t}(s) d s=\int_{-\infty}^{t} U_{f_{t}}(0, s-t) \gamma(s) d s=\int_{-\infty}^{t} U_{f}(t, s) \gamma(s) d s,
$$

and from (5.15) it follows that $\tilde{b}\left(f_{t}\right)=y(t, f, b(f))$. Thus, $b(f)$ is a continuous equilibrium for (5.5) because $b\left(f_{t}\right)(s)=\widetilde{b}\left(f_{t+s}\right)=y(t+s, f, b(f))$ for $s \in[-1,0]$, i.e. $\left.b\left(f_{t}\right)=y_{t}(\cdot, f, b(f))\right)$, as stated. In addition, from (5.7) it follows that this 
equilibrium $b(f)$ is globally exponentially stable for $\Psi$ in (5.5). Finally notice that from (A2), again a comparison result provides $x_{t}(\cdot, f, b(f)) \leqslant y_{t}(\cdot, f, b(f))=b\left(f_{t}\right)$ for $t \geqslant 0$, and we conclude that $b(f)$ is a continuous super-equilibrium for (5.4), which finishes the proof of (ii).

We will omit the proofs of (iii) and (iv) because follow similar arguments to those of (i) and (ii). We want only to remark the following differences. Notice that $Z_{f}(t, s):=\exp \left(-\int_{s}^{t} \alpha(u) d u\right)$ is the fundamental matrix of the Carathéodory ordinary differential equation $z^{\prime}(t)=-\alpha(t) z(t)$ with $Z_{f}(s, s)=1, U_{f}(t, s)$ is the fundamental matrix of (5.3), and from (A2) and (5.7) we also deduce that $Z_{f}(t, s) \leqslant$ $U_{f}(t, s) \leqslant K e^{-\delta(t-s)}$ whenever $s \leqslant t$. Moreover, in (i) and (ii) we were comparing the solutions of equations (5.1) and (5.2), i.e. $x(t, f, \phi) \leqslant y(t, f, \phi)$, so that it is said that (5.2) is a majorant of (5.1), and now again from (A1) and (A2), the Carathéodory ordinary differential equation $y^{\prime}(t)=-\alpha(t) y(t)+h_{0}(t)$ is a minorant of (5.1). This is the reason why now $a(f)$, which is a globally exponentially stable continuous equilibrium for $\Psi$ in (5.5), is a continuous sub-equilibrium for (5.4).

Finally, $a(f) \leqslant b(f)$ for each $f \in E$ follows from the above comparison of solutions, and $0 \ll a(f)$ for each $f \in E$ is a consequence of the definition of $a(f)$ and the belonging of $h_{0}$ to the set $E_{1}$, which is closed, invariant and does not contain the null function, from which it can be shown that the set $\left\{r \in[-\infty, u] \mid h_{0}(r)>0\right\}$ have positive measure for each $u \in[-1,0]$.

As a consequence, from Theorem 4.4 we deduce the existence of two equilibria for the skew-product semiflow (5.4) induced by the family of equations (5.1), one is lower-semicontinuous $u: E \rightarrow \mathcal{C}^{+}, f \mapsto u(f)$, the other is upper-semicontinuous $v: E \rightarrow \mathcal{C}^{+}, f \mapsto v(f)$, and they satisfy $0 \ll u(f) \leqslant v(f)$. It can be shown that they define the top and lower covers of a pullback attractor for the evolution processes induced by (5.1) (we refer the reader to Kloeden and Rasmussen [10] for these concepts).

In addition, we assume the following condition implying the sublinearity of the skew-product semiflow:

(A4) for each function $f=(h, \alpha, \beta, \gamma) \in E$, the function $h$ is sublinear, that is, for each $y \in \mathbb{R}^{+}$and $\lambda \in[0,1], h(t, \lambda y) \geqslant \lambda h(t, y)$ for a.e. $t \in \mathbb{R}$.

From this, we deduce that $g(t, x, y)=-\alpha(t) x+h(t, y)$ is also sublinear, i.e. satisfies $(\mathbf{S})$, and hence the skew-product semiflow (5.4) is sublinear, as shown in Proposition 4.8. Next lemma characterizes the points of strong sublinearity for the skew-product semiflow (5.4) (see (4.5) for the definition).

Lemma 5.3. Consider $f=(h, \alpha, \beta, \gamma) \in E$ and assume that the scalar function $h$ satisfies the following property of strong sublinearity:

- for each $\delta>0$ the set of points $t \in(0, \delta)$ such that

$$
h(t, \lambda y)>\lambda h(t, y) \quad \text { for each } y>0 \text { and } \lambda \in(0,1)
$$

has positive Lebesgue measure.

Then, $f$ is a point of strong sublinearity for the skew-product semiflow (5.4).

Proof. We will just provide a sketch of the proof. First, notice that the function $g(t, x, y)=-\alpha(t) x+h(t, y)$ satisfies the same property of strong sublinearity on $x$ and $y$. From this, reasoning as in Proposition 4.8 to transform the delay equation (5.1) into an ordinary one in $[0,1]$, we check condition (ii) of [28, Theorem 4] 
to deduce that $x(t, f, \lambda \phi)>\lambda x(t, f, \phi)$ for each $t \in(0,1], \lambda \in(0,1)$ and $\phi \gg 0$. In a recursive way we can check that this also holds for $t>0$, which means that $x_{t}(\cdot, f, \lambda \phi) \gg \lambda x_{t}(\cdot, f, \phi)$ for $t>1$, as claimed.

As a consequence, under assumptions (A1)-(A4), we can apply the conclusions of Theorem 4.10 to the monotone, sublinear and continuous skew-product semiflow (5.4), characterizing the points of strong sublinearity on $E_{-}$and $E_{+}$, as in the previous lemma. Thus, the existence of a unique continuous equilibrium whose graph coincides with the pullback attractor of the equation is shown.

5.2. Carathéodory non-autonomous cyclic feedback system. We finish with an application of our previous results to the mathematical model of biochemical feedback in protein synthesis given by the system of Carathéodory differential equations

$$
\begin{aligned}
x_{1}^{\prime}(t) & =h\left(t, x_{m}(t)\right)-\alpha_{1}(t) x_{1}(t), \\
x_{i}^{\prime}(t) & =x_{i-1}(t)-\alpha_{i}(t) x_{i}(t), \quad \text { for } 2 \leqslant i \leqslant m .
\end{aligned}
$$

The system (5.16) expresses a model for a biochemical control circuit in which each of the $x_{j}$ represents the concentration of an enzyme; hence $x_{j} \geqslant 0$ for $j=1, \ldots, m$. The autonomous ordinary case was firstly introduced by Selgrade [23]. Different extensions to the periodic and the autonomous functional cases are explored in Smith [26], Krause and Ranft [12], Smith and Thieme [27], and references therein. Chueshov [6] analyzes the random case and Novo et al. [21] the ordinary deterministic non-autonomous case. The case of finite-delay was considered, for the concave case, in Novo et al. [19], and, for the sublinear case, in Novo and Obaya [20].

We state the assumptions to be considered for this problem, concerning with the Carathéodory ordinary case. Notice that (B1) coincides with (A1) but we repeat it by completeness.

(B1) $E_{1}$ is a closed invariant and bounded subset of functions $\alpha \in L_{l o c}^{1}$ such that $\alpha(t) \geqslant 0$ for a.e. $t \in \mathbb{R}$ and the null function $\alpha=0$ does not belong to $E_{1}$. Let $D$ be a countable dense subset of $\mathbb{R}$ and consider the subset of $\mathfrak{L} \mathfrak{C}$ given by

$$
E_{2}=\left\{\begin{array}{l|l}
h \in \mathfrak{L} \mathfrak{C} & \begin{array}{l}
h \text { satisfies }\left(\mathbf{K}_{2}\right), h_{0}(t):=h(t, 0) \in E_{1} \\
\text { and } h(t, y)=h(t, 0) \text { for } y \leqslant 0
\end{array}
\end{array}\right\} .
$$

Notice that $E_{2}$ is invariant and closed with respect to the topology $\mathcal{T}_{D}$.

(B2) $E$ is a subset of

$$
\left\{\begin{array}{l|l}
f=\left(h, \alpha_{1}, \ldots, \alpha_{m}, \beta, \gamma\right) \mid \begin{array}{l}
h \in E_{2}, \alpha_{1}, \ldots, \alpha_{m}, \beta, \gamma \in E_{1} \text { and } \\
h(t, y) \leqslant \beta(t) y+\gamma(t), \forall y \in \mathbb{R}, \text { a.e. } t \in \mathbb{R}
\end{array}
\end{array}\right\}
$$

which is invariant and closed for the product topology.

(B3) There are constants $K, \delta>0$ such that for each $f=\left(\alpha_{1}, \ldots, \alpha_{m}, \beta, \gamma\right) \in E$

$$
\|U(t, f)\| \leqslant K e^{-\delta t} \quad \text { for each } t>0,
$$

where $U(t, f)$ is the fundamental matrix solution of the system

$$
\begin{aligned}
& z_{1}^{\prime}(t)=\beta(t) z_{m}(t)-\alpha_{1}(t) z_{1}(t), \\
& z_{i}^{\prime}(t)=z_{i-1}(t)-\alpha_{i}(t) z_{i}(t), \quad \text { for } 2 \leqslant i \leqslant m,
\end{aligned}
$$

principal at $t=0$, that is, $U(0, f)=I_{m}$. 
Under these assumptions, the function defining (5.16) satisfies conditions $\left(\mathbf{K}_{1}\right)$ and $\left(\mathbf{L}_{1}\right)$, and from [28, Theorem 2] and Proposition 3.20, the skew-product semiflow

$$
\Phi: \mathbb{R}^{+} \times E \times\left(\mathbb{R}^{+}\right)^{m} \rightarrow E \times\left(\mathbb{R}^{+}\right)^{m}, \quad\left(t, f, x_{0}\right) \mapsto\left(f_{t}, x\left(t, f, x_{0}\right)\right),
$$

is monotone and continuous for the above product topology.

Remark 5.4. If we denote by $U_{f}(t, s)$ the fundamental matrix solution for (5.17) principal at $t=s$, that is, $U_{f}(s, s)=I_{m}$, from assumption (B3) we deduce that

$$
\left\|U_{f}(t, s)\right\| \leqslant K e^{-\delta(t-s)} \quad \text { whenever } t \geqslant s,
$$

because $U_{f}(t, s)=U\left(t-s, f_{s}\right)$. Moreover, denoting by $Z_{f}(s, t)$ the fundamental matrix solution of

$$
\begin{aligned}
& z_{1}^{\prime}(t)=-\alpha_{1}(t) z_{1}(t), \\
& z_{i}^{\prime}(t)=z_{i-1}(t)-\alpha_{i}(t) z_{i}(t), \quad \text { for } 2 \leqslant i \leqslant m,
\end{aligned}
$$

and since, from (B1), the system (5.19) is a minorant of (5.17), we also have

$$
\left\|Z_{f}(t, s)\right\| \leqslant K e^{-\delta(t-s)} \quad \text { whenever } t \geqslant s .
$$

In addition, notice that if the first component of $z_{0}$ is positive, i.e. $\left(z_{0}\right)_{1}>0$, the solution of (5.19) with this initial data $z_{0}$ is strongly positive, that is, $Z_{f}(t, 0) z_{0} \gg 0$ for each $t>0$. The reason is as follows. From $z_{1}^{\prime}(t)=-\alpha_{1}(t) z_{1}(t)$ we deduce that $\left(Z_{f}(t, 0) z_{0}\right)_{1}=\left(\int_{0}^{t} \exp \left(-\alpha_{1}(r)\right) d r\right)\left(z_{0}\right)_{1}>0$ for each $t \geqslant 0$. For the second component, from $z_{2}^{\prime}(t)=z_{1}(t)-\alpha_{2}(t) z_{2}(t)>-\alpha_{2}(t) z_{2}(t)$ and comparison of solutions we deduce that $\left(Z_{f}(t, 0) z_{0}\right)_{2}>\left(\int_{0}^{t} \exp \left(-\alpha_{2}(r)\right) d r\right)\left(z_{0}\right)_{2} \geqslant 0$ for each $t>0$, and the same reasoning for the rest of the components provides $Z_{f}(t, 0) z_{0} \gg 0$ for each $t>0$, as claimed.

Proposition 5.5. Under assumptions (B1)-(B3) and notation of Remark 5.4, consider the unit vector $\boldsymbol{e}_{1}=(1,0, \ldots, 0)^{t}$. Then

(i) $b: E \rightarrow\left(\mathbb{R}^{+}\right)^{m}, f \mapsto b(f)=\int_{-\infty}^{0} U_{f}(0, s) \gamma(s) \mathbf{e}_{1} d s$ is a continuous superequilibrium for (5.18),

(ii) $a: E \rightarrow\left(\mathbb{R}^{+}\right)^{m}, f \mapsto a(f)=\int_{-\infty}^{0} Z_{f}(0, s) h_{0}(s) \mathbf{e}_{1} d s$, with $h_{0}$ defined in (B1), is a continuous sub-equilibrium for (5.18),

and they satisfy $0 \ll a(f) \leqslant b(f)$.

Proof. We omit the proof because it is similar to that of Proposition 5.2. However, we want to remark the following facts. Although we introduced the definitions of sub and super-equilibria for skew-product semiflows induced by Carathéodory delay differential equations, they are easily adapted to Carathéodory ordinary differential equations, changing $\mathcal{C}=C\left([-1,0], \mathbb{R}^{N}\right)$ by $\mathbb{R}^{m}$ in our case.

We want also to notice that in this example, the system

$$
\begin{array}{ll}
z_{1}^{\prime}(t)=\beta(t) z_{m}(t)-\alpha_{1}(t) z_{1}(t)+\gamma(t), & \\
z_{i}^{\prime}(t)=z_{i-1}(t)-\alpha_{i}(t) z_{i}(t), & \text { for } 2 \leqslant i \leqslant m
\end{array}
$$


is a majorant for (5.16) in the sense explained in the proof of Proposition 5.2, and $z(t)=\int_{-\infty}^{t} U_{f}(0, s) \gamma(s) \mathbf{e}_{1} d s$ is a globally defined bounded solution for it. Analogously, $z(t)=\int_{-\infty}^{t} Z_{f}(0, s) h_{0}(s) \mathbf{e}_{1} d s$ is a globally defined bounded solution of

$$
\begin{aligned}
& z_{1}^{\prime}(t)=-\alpha_{1}(t) z_{1}(t)+h_{0}(t), \\
& z_{i}^{\prime}(t)=z_{i-1}(t)-\alpha_{i}(t) z_{i}(t), \quad \text { for } 2 \leqslant i \leqslant m,
\end{aligned}
$$

which is a minorant of (5.16) because $h$ satisfies $\left(\mathbf{K}_{1}\right)$, and then $h(t, z) \geqslant h(t, 0)=$ $h_{0}(t)$ for $z \geqslant 0$. These two facts are in this case the main ingredients for the proof of (i), (ii) and $a(f) \leqslant b(f)$ for each $f \in E$. The proof of $0 \ll a(f)$ for each $f \in E$ relies now on the belonging of $h_{0}$ to $E_{1}$, so that $\left\{s \in(-\infty, 0] \mid h_{0}(s)>0\right\}$ has positive measure, and then for all these points, the first component of $h_{0}(s) \mathbf{e}_{1}$ is positive and $Z_{f}(0, s) h_{0}(s) \mathbf{e}_{1}=Z_{f_{s}}(-s, 0) h_{0}(s) \mathbf{e}_{1} \gg 0$, as shown in Remark 5.4.

As a in the previous scalar example, from Theorem 4.4 we deduce the existence of two equilibria for the skew-product semiflow (5.18) induced by the family of systems (5.16). One of them, $u: E \rightarrow \mathcal{C}^{+}, f \mapsto u(f)$ is lower-semicontinuous, the other, $v: E \rightarrow \mathcal{C}^{+}, f \mapsto v(f)$, is upper-semicontinuous, and they satisfy $0 \ll$ $u(f) \leqslant v(f)$. They define the top and lower covers of a pullback attractor for the skew-product semiflow (5.18).

We finish with the assumption of a sublinearity condition for this case, in order to apply the conclusions of Theorem 4.10.

(B4) For each $f=\left(h, \alpha_{1}, \ldots, \alpha_{m}, \beta, \gamma\right) \in E$, the function $h$ is sublinear, that is, for each $y \in \mathbb{R}^{+}$and $\lambda \in[0,1], h(t, \lambda y) \geqslant \lambda h(t, y)$ for a.e. $t \in \mathbb{R}$.

From this we deduce that the function $g: \mathbb{R} \times\left(\mathbb{R}^{+}\right)^{m} \rightarrow\left(\mathbb{R}^{+}\right)^{m}$ of system (5.16) defined as $g_{1}(t, x)=h\left(t, x_{m}\right)-\alpha_{1}(t) x_{1}$ and $g_{i}(t, x)=x_{i-1}-\alpha_{i}(t) x_{i}$ for $2 \leqslant i \leqslant m$ is sublinear, and hence the skew-product semiflow (5.18) is sublinear.

As in (4.5), but now for the case of ordinary differential equations, $f$ is a point of strong sublinearity for the skew-product semiflow (5.18) if

$$
x\left(t, f, \lambda x_{0}\right) \gg \lambda x\left(t, f, x_{0}\right) \quad \text { whenever } t>0, \lambda \in(0,1) \text { and } x_{0} \gg 0 .
$$

Next lemma provides a characterization for these points of strong sublinearity.

Lemma 5.6. Consider $f=\left(h, \alpha_{1}, \ldots, \alpha_{m}, \beta, \gamma\right) \in E$ and assume that the scalar function $h$ satisfies the following property of strong sublinearity:

- for each $\delta>0$ the set of points $t \in(0, \delta)$ such that

$$
h(t, \lambda y)>\lambda h(t, y) \quad \text { for each } y>0 \text { and } \lambda \in(0,1)
$$

has positive Lebesgue measure.

Then, $f$ is a point of strong sublinearity for the skew-product semiflow (5.18).

Proof. Take $\lambda \in(0,1), x_{0} \gg 0, v(t)=\lambda x\left(t, f, x_{0}\right)$ and $w(t)=x\left(t, f, \lambda x_{0}\right)$. Since the skew-product semiflow (5.18) is sublinear we know that $v(t) \leqslant w(t)$ for each $t \geqslant 0$. Moreover, consider the scalar linear equation

$$
y^{\prime}(t)=l_{1}(t, y(t))=h\left(t, \lambda x_{m}\left(t, f, x_{0}\right)\right)-\alpha_{1}(t) y(t) .
$$

From (5.21) and $v_{1}(t) \leqslant w_{1}(t)$, we deduce that for each $\delta>0$

$v_{1}^{\prime}(t)-l_{1}\left(t, v_{1}(t)\right)=\lambda h\left(t, x_{m}\left(t, f, x_{0}\right)\right)-h\left(t, \lambda x_{m}\left(t, f, x_{0}\right)\right)<0=w_{1}^{\prime}(t)-l_{1}\left(t, w_{1}(t)\right)$

for $t$ in a subset of $(0, \delta)$ with positive Lebesgue measure. Therefore, condition (ii) of [28, Theorem 4] holds and we conclude that $v_{1}(t)<w_{1}(t)$ for each $t>0$. 
Next, we take $y^{\prime}(t)=l_{2}(t, y(t))=x_{1}\left(t, f, \lambda x_{0}\right)-\alpha_{2}(t) y(t)$. As before, but now from $v_{1}(t)<w_{1}(t)$ for each $t>0$ and $v_{2}(t) \leqslant w_{2}(t)$ we obtain

$$
v_{2}^{\prime}(t)-l_{2}\left(t, v_{2}(t)\right)<0=w_{2}^{\prime}(t)-l_{2}\left(t, w_{2}(t)\right),
$$

for each $t>0$, which implies $v_{2}(t)<w_{2}(t)$ for each $t>0$. The inequalities for the rest of the components are obtained in a similar way, so that $v(t)<w(t)$ for each $t>0$, that is, inequality (5.20) holds, and $f$ is a point of strong sublinearity for (5.18), as claimed.

As a consequence, under assumptions (B1)-(B4), we can apply the conclusions of Theorem 4.10 to the monotone, sublinear and continuous skew-product semiflow (5.18), characterizing the points of strong sublinearity on $E_{-}$and $E_{+}$, as in the previous lemma. Therefore, the existence of a unique continuous equilibrium whose graph coincides with the pullback attractor of the system is shown.

\section{REFERENCES}

[1] Z. Artstein, Topological dynamics of an ordinary differential equation, J. Differential Equations 23 (1977), 216-223.

[2] Z. ARTstein, Topological dynamics of ordinary differential equations and Kurzweil equations, J. Differential Equations 23 (1977), 224-243.

[3] Z. Artstein, The limiting equations of nonautonomous ordinary differential equations, $J$. Differential Equations 25 (1977), 184-202.

[4] J-P. Aubin, H. Frankowska: Set-valued analysis, Birkhäuser Basel, 1990.

[5] F. Brauer, C. Castillo-Chávez: Mathematical Models in Population Biology and Epidemiology, Springer New York Dordrecht Heidelberg London, 2012.

[6] I.D. Chueshov, Monotone Random Systems. Theory and Applications, Lecture Notes in Math. 1779, Springer-Verlag, Berlin, Heidelberg, 2002.

[7] E.A. Coddington, N. Levinson, Theory Of Ordinary Differential Equations, McGraw-Hill, New York, 1955.

[8] J.K. Hale, Theory Of Functional Differential Equations, Springer-Verlag, New York, 1977.

[9] A.J. Heunis: Continuous dependence of the solutions of an ordinary differential equation, $J$. Differential Equations 54 (1984), 121-138.

[10] P. Kloeden, M. Rassmussen, Nonautonomous Dynamical Systems, Mathematical Surveys and Monographs, Amer. Math. Soc., 2011.

[11] U. Krause, R.D. Nussbaum, A limit set trichotomy for self-mappings of normal cones in Banach spaces, Nonlinear Anal. 20 (1993), no. 7, 855-870.

[12] U. Krause, P. Ranft, A limit set trichotomy for monotone nonlinear dynamical systems. Nonlinear Anal. 19 (1992), 375-392.

[13] I.P. Longo, S. Novo, R. Obaya, Topologies of $L_{l o c}^{p}$ type for Carathéodory functions with applications in non-autonomous differential equations, J. Differential Equations, 263 (2017), $7187-7220$.

[14] I.P. Longo, S. Novo, R. Obaya, Weak topologies for Carathéodory differential equations. Continuous dependence, exponential dichotomy and attractors, J. Dynam. Differential Equations 31, no. 3 (2019), 1617-1651.

[15] I.P. Longo, S. Novo, R. ObaYA, Topologies of continuity for Carathéodory delay differential equations with applications in non-autonomous dynamics, Discrete Contin. Dyn. Sist. 39, no. 9 (2019), 5491-5520.

[16] R.K. Miller, G. Sell, Volterra Integral Equations and Topological Dynamics, Mem. Amer. Math. Soc., no. 102, Amer. Math. Soc., Providence, 1970.

[17] R.K. Miller, G. Sell, Existence, uniqueness and continuity of solutions of integral equations, Ann. Math. Pura Appl. 80 (1968), 135-152; Addendum: ibid. 87 (1970), 281-286.

[18] L.W. Neustadt, On the solutions of certain integral-like operator equations. Existence, uniqueness and dependence theorems, Arch. Rational Mech. Anal. 38 (1970), 131-160.

[19] S. Novo, C. NúÑEz, R. OBAYA, Almost automorphic and almost periodic dynamics for quasimonotone non-autonomous functional differential equations, J. Dynam. Differential Equations 17 no. 3 (2005) 589-619. 
[20] S. Novo, R. OваYA, Non-autonomous functional differential equations and applications, Stability and bifurcation theory for non-autonomous differential equations, Lecture Notes in Math., vol. 2065, Springer, Heidelberg (2013), 185-263,

[21] S. Novo, R. Obaya, A.M. Sanz, Attractor minimal sets for cooperative and strongly convex delay differential systems. J. Differential Equations 208 (1) (2005), 86-123.

[22] Z. OpIAL: Continuous parameter dependence in linear systems of differential equations. $J$. Differential Equations 3 (1967), 571-579.

[23] J.F. Selgrade, Asymptotic behavior of solutions to single loop positive feedback systems. J. Differential Equations 38 (1980), 80-103.

[24] G. SEll: Topological Dynamics and Ordinary Differential Equations, Van NostrandReinhold, London, 1971.

[25] H.L. Sмith, An Introduction to Delay Differential Equations with Applications to the Life Sciences. Springer-Verlag New York, 2011.

[26] H.L. Sмith, Monotone Dynamical Systems. An introduction to the Theory of Competitive and Cooperative Systems. Amer. Math. Soc., Providence, 1995.

[27] H.L. Smith, H.R. Thieme, Strongly order preserving semiflows generated by functional differential equations. J. Differential Equations 93 (2) (1991), 332-363.

[28] W. Walter, On strongly monotone flows, Ann. Polon. Math. 66 (1997), 269-274.

[29] X.Q. ZhaO: Global attractivity in monotone and subhomogeneous almost periodic systems. J. Differential Equations 187 (2) (2003), 494-509.

E-mail address, Iacopo P. Longo: longoi@ma.tum.de

E-mail address, Sylvia Novo: sylnov@wmatem.eis.uva.es

E-mail address, Rafael Obaya: rafoba@wmatem.eis.uva.es

(Iacopo P. Longo) Technische Universität München, Forschungseinheit Dynamics, Zentrum Mathematik, M8, Boltzmannstrasse 3, 85748 Garching bei München, Germany.

(S. Novo and R. Obaya) Departamento de Matemática Aplicada, Universidad de Valladolid, Paseo del Cauce 59, 47011 Valladolid, Spain. 